DOI 10.4171/JEMS/252

Jiří Matoušek · Martin Tancer · Uli Wagner

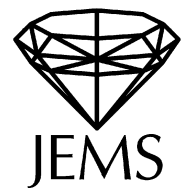

\title{
Hardness of embedding simplicial complexes in $\mathbb{R}^{d}$
}

Received August 26, 2008 and in revised form April 22, 2009

\begin{abstract}
Let $\mathrm{EMBED}_{k \rightarrow d}$ be the following algorithmic problem: Given a finite simplicial complex $K$ of dimension at most $k$, does there exist a (piecewise linear) embedding of $K$ into $\mathbb{R}^{d}$ ? Known results easily imply the polynomiality of $\operatorname{EMBED}_{k \rightarrow 2}(k=1,2$; the case $k=1, d=2$ is graph planarity) and of $\mathrm{EMBED}_{k \rightarrow 2 k}$ for all $k \geq 3$.

We show that the celebrated result of Novikov on the algorithmic unsolvability of recognizing the 5-sphere implies that $\operatorname{EMBED}_{d \rightarrow d}$ and $\operatorname{EMBED}_{(d-1) \rightarrow d}$ are undecidable for each $d \geq 5$. Our main result is the NP-hardness of $\mathrm{EMBED}_{2 \rightarrow 4}$ and, more generally, of $\mathrm{EMBED}_{k \rightarrow d}$ for all $k, d$ with $d \geq 4$ and $d \geq k \geq(2 d-2) / 3$. These dimensions fall outside the metastable range of a theorem of Haefliger and Weber, which characterizes embeddability using the deleted product obstruction. Our reductions are based on examples, due to Segal, Spież, Freedman, Krushkal, Teichner, and Skopenkov, showing that outside the metastable range the deleted product obstruction is not sufficient to characterize embeddability.
\end{abstract}

\section{Introduction}

Does a given (finite) simplicial complex $1 / K$ of dimension at most $k$ admit an embedding into $\mathbb{R}^{d}$ ? We consider the computational complexity ${ }^{2}$ of this question, regarding $k$ and $d$ as fixed integers. To our surprise, apparently this question has not been explicitly addressed before (with the exception of $k=1, d=2$ which is graph planarity), as far we

J. Matoušek, M. Tancer: Department of Applied Mathematics and Institute of Theoretical Computer Science (ITI), Charles University, Malostranské nám. 25, 11800 Praha 1, Czech Republic; Institute of Theoretical Computer Science, ETH Zürich, 8092 Zürich, Switzerland;

e-mail: matousek@kam.mff.cuni.cz, tancer@kam.mff.cuni.cz

U. Wagner: Institute of Theoretical Computer Science, ETH Zürich, 8092 Zürich, Switzerland; e-mail: wagneru@inf.ethz.ch

1 We assume that the reader is somewhat familiar with basic notions of combinatorial topology (introductory chapters of books like [Mun84, Hat01, Mat03] should provide a sufficient background). Terminology and basic facts concerning simplicial complexes will be recalled in Section 2 Later on, in some of the proofs, we will need other, slightly more advanced topological notions and results, which would take too much space to define properly. We hope that the main ideas can be followed even when such things are skipped.

2 For basic definitions from computational complexity, such as polynomial-time algorithm, NPhardness, reduction, or 3-SAT the reader can refer to any introductory textbook on algorithms. Very recent textbooks were written by Arora and Barak [AB09] and by Goldreich [Gol08]. We also highly recommend Wigderson's essay [Wig07]. 
could find. Besides its intrinsic interest for the theory of computing, an algorithmic view of a classical subject such as embeddability may lead to new questions and also to a better understanding of known results. For example, computational complexity can be seen as a concrete "measuring rod" that allows one to compare the "relative strength" of various embeddability criteria, respectively of examples showing the necessity of dimension restrictions in the criteria. Moreover, hardness results provide concrete evidence that for a certain range of parameters (outside the so-called metastable range), no simple structural characterization of embeddability (such as Kuratowski's forbidden minor criterion for graph planarity) is to be expected.

For algorithmic embeddability problems, we consider piecewise linear (PL) embeddings. Let us remark that there are at least two other natural notions of embeddings of simplicial complexes in $\mathbb{R}^{d}$ : linear embeddings (also called geometric realizations), which are more restrictive than PL embeddings, and topological embeddings, which give us more freedom than PL embeddings. We will recall the definitions in Section 2, here we quickly illustrate the differences with a familiar example: embeddings of 1-dimensional simplicial complexes, or simple graphs, into $\mathbb{R}^{2}$. For a topological embedding, the image of each edge can be an arbitrary (curved) Jordan arc, for a PL embedding it has to be a polygonal arc (made of finitely many straight line segments), and for a linear embedding, it must be a single straight line segment. For this particular case $(k=1, d=2)$, all three notions happen to give the same class of embeddable complexes, namely, all planar graphs (by Fáry's theorem). For higher dimensions there are significant differences, though, which we also discuss in Section 2 .

Here we are mainly interested in embeddability in the topological sense (as opposed to linear embeddability, which is a much more geometric problem and one with a very different flavor), but since it seems problematic to deal with arbitrary topological embeddings effectively, we stick to PL embeddings, which can easily be represented in a computer.

We thus introduce the decision problem $\mathrm{EMBED}_{k \rightarrow d}$, whose input is a simplicial complex $K$ of dimension at most $k$, and where the output should be YES or NO depending on whether $K$ admits a PL embedding into $\mathbb{R}^{d}$.

We assume $k \leq d$, since a $k$-simplex cannot be embedded in $\mathbb{R}^{k-1}$. For $d \geq 2 k+1$ the problem becomes trivial, since it is well known that every finite $k$-dimensional simplicial complex embeds in $\mathbb{R}^{2 k+1}$, even linearly (this result goes back to Menger). In all other cases, i.e., $k \leq d \leq 2 k$, there are both YES and NO instances; for the NO instances one can use, e.g., examples of $k$-dimensional complexes not embeddable in $\mathbb{R}^{2 k}$ due to Van Kampen [vK32] and Flores [Flo34].

Let us also note that the complexity of this problem is monotone in $k$ by definition, since an algorithm for $\mathrm{EMBED}_{k \rightarrow d}$ also solves $\mathrm{EMBED}_{k^{\prime} \rightarrow d}$ for all $k^{\prime} \leq k$.

Tractable cases. It is well known that $\mathrm{EMBED}_{1 \rightarrow 2}$ (graph planarity) is linear-time solvable [HT74]. Based on planarity algorithms and on a characterization of complexes embeddable in $\mathbb{R}^{2}$ due to Halin and Jung [HJ64], it is not hard to come up with a linear-time decision algorithm for $\mathrm{EMBED}_{2 \rightarrow 2}$. Since we do not know of a reference, we outline such an algorithm in Appendix A Daniel Král' (personal communication) has independently devised a linear-time algorithm that actually produces an embedding. 
There are many problems in computational topology that are easy for low dimensions (say up to dimension 2 or 3 ) and become intractable from some dimension on (say 4 or 5); we mention some of them later. For the embeddability problem, the situation is subtler, since there are tractable cases in arbitrarily high dimensions, namely, $\mathrm{EMBED}_{k \rightarrow 2 k}$ for every $k \geq 3$.

The algorithm is based on ideas of Van Kampen [vK32], which were made precise by Shapiro [Sha57] and independently by Wu [Wu65]. Since we are not aware of any treatment in an algorithmic context, and since some of the published descriptions have a small flaw (a sign error) and others use a somewhat advanced language of algebraic topology, we give a self-contained elementary presentation of the algorithm (but not a proof of correctness) in Appendix D

Hardness. According to a celebrated result of Novikov ([VKF74]; also see, e.g., [Nab95] for an exposition), the following problem is algorithmically unsolvable: Given a $d$-dimensional simplicial complex, $d \geq 5$, decide whether it is homeomorphic to $S^{d}$, the $d$ dimensional sphere. By a simple reduction we obtain the following result:

Theorem 1.1. $\operatorname{EMBED}_{(d-1) \rightarrow d}$ (and hence also $\mathrm{EMBED}_{d \rightarrow d}$ ) is algorithmically undecidable for every $d \geq 5$.

This has an interesting consequence, which in some sense strengthens results of Brehm and Sarkaria [BS92]:

Corollary 1.2. For every computable (recursive) function $f: \mathbb{N} \rightarrow \mathbb{N}$ and for every $d \geq 5$ there exist $n$ and a finite $(d-1)$-dimensional simplicial complex $K$ with $n$ simplices that PL-embeds in $\mathbb{R}^{d}$ but such that no subdivision of $K$ with at most $f(n)$ simplices embeds linearly in $\mathbb{R}^{d}$.

Our main result is hardness for cases where $d \geq 4$ and $k$ is larger than roughly $\frac{2}{3} d$.

Theorem 1.3. EMBED $_{k \rightarrow d}$ is NP-hard for every pair $(k, d)$ with $d \geq 4$ and $d \geq k \geq$ $(2 d-2) / 3$.

We prove a special case of this theorem, the NP-hardness of $\mathrm{EMBED}_{2 \rightarrow 4}$, in Section 4 , the proof is somewhat more intuitive than for the general case and it contains most of the ideas. All the remaining cases are proved in Section 5 .

Let us briefly mention where the dimension restriction $k \geq(2 d-2) / 3$ comes from. There is a certain necessary condition for embeddability of a simplicial complex into $\mathbb{R}^{d}$, called the deleted product obstruction. A celebrated theorem of Haefliger and Weber, which is a far-reaching generalization of the ideas of Van Kampen mentioned above, asserts that this condition is also sufficient provided that $k \leq \frac{2}{3} d-1$ (these $k$ are said to lie in the metastable range). The condition on $k$ in Theorem 1.3 is exactly that $k$ must be outside of the metastable range (we refer to Appendix $B$ for a brief discussion and references).

There are examples showing that the restriction to the metastable range in the Haefliger-Weber theorem is indeed necessary, in the sense that whenever $d \geq 3$ and $d \geq$ 
$k>(2 d-3) / 3$, there are $k$-dimensional complexes that cannot be embedded into $\mathbb{R}^{d}$ but the deleted product obstruction fails to detect this. We use constructions of this kind, namely, examples due to Segal and Spież [SS92], Freedman, Krushkal, and Teichner [FKT94], and Segal, Skopenkov, and Spież [SSS98], as the main ingredient in our proof of Theorem 1.3 .

\begin{tabular}{c|ccccccccccccc}
$k=$ & 2 & 3 & 4 & 5 & 6 & 7 & 8 & 9 & 10 & 11 & 12 & 13 & 14 \\
\hline 1 & $\mathrm{P}$ & + & + & + & + & + & + & + & + & + & + & + & + \\
2 & $\mathrm{P}$ & $?$ & $\mathrm{NPh}$ & + & + & + & + & + & + & + & + & + & + \\
3 & & $?$ & $\mathrm{NPh}$ & $\mathrm{NPh}$ & $\mathrm{P}$ & + & + & + & + & + & + & + & + \\
4 & & & $\mathrm{NPh}$ & $\mathrm{UND}$ & $\mathrm{NPh}$ & $\mathrm{NPh}$ & $\mathrm{P}$ & + & + & + & + & + & + \\
5 & & & & UND & UND & NPh & NPh & $?$ & $\mathrm{P}$ & + & + & + & + \\
6 & & & & & UND & UND & NPh & NPh & NPh & $?$ & $\mathrm{P}$ & + & + \\
7 & & & & & & UND & UND & NPh & NPh & NPh & $?$ & $?$ & P
\end{tabular}

Table 1. The complexity of $\mathrm{EMBED}_{k \rightarrow d}(\mathrm{P}=$ polynomial-time solvable, $\mathrm{UND}=$ algorithmically undecidable, $\mathrm{NPh}=\mathrm{NP}$-hard, $+=$ always embeddable, $?=$ no result known).

Discussion. The current complexity status of $\mathrm{EMBED}_{k \rightarrow d}$ is summarized in Table 1 In our opinion, the most interesting currently open cases are $(k, d)=(2,3)$ and $(3,3)$.

These are outside the metastable range, and it took the longest to find an example showing that they are not characterized by the deleted product obstruction; see [GS06]. That example does not seem to lend itself easily to a hardness reduction, though.

A variation on the proof of our undecidability result (Theorem 1.1) shows that both $\mathrm{EMBED}_{2 \rightarrow 3}$ and $\mathrm{EMBED}_{3 \rightarrow 3}$ are at least as hard as the problem of recognizing the 3sphere (that is, given a simplicial complex, decide whether it is homeomorphic to $S^{3}$ ). The latter problem is in NP [Iva08, Sch04], but no hardness result seems to be known.

For the remaining question marks in the table (with $d \geq 9$ ), which all lie in the metastable range, it seems that existing tools of algebraic topology, such as Postnikov towers and/or suitable spectral sequences, could lead at least to decision algorithms, or even to polynomial-time algorithms in some cases. Here the methods of "constructive algebraic topology" mentioned below, which imply, e.g., the computability of higher homotopy groups, should be relevant. However, as is discussed, e.g., in [RRS06], computability issues in this area are often subtle, even for questions considered well understood in classical algebraic topology. We hope to clarify these things in a future work.

Our NP-hardness results are probably not the final word on the computational complexity of the corresponding embeddability problems; for example, some or all of these might turn out to be undecidable.

Related work. Among the most important computational problems in topology are the homeomorphism problem for manifolds, and the equivalence problem for knots. The first one asks whether two given manifolds $M_{1}$ and $M_{2}$ (given as simplicial complexes, say) are homeomorphic. The second one asks whether two given knots, i.e., PL embeddings $f, g: S^{1} \rightarrow \mathbb{R}^{3}$, are equivalent, i.e., whether there is a PL homeomorphism $h: \mathbb{R}^{3} \rightarrow \mathbb{R}^{3}$ such that $f=h \circ g$. An important special case of the latter is the knot triviality problem: Is a given knot equivalent to the trivial knot (i.e., the standard geometric circle placed in $\left.\mathbb{R}^{3}\right)$ ? 
There is a vast amount of literature on computational problems for 3-manifolds and knots. For instance, it is algorithmically decidable whether a given 3-manifold is homeomorphic to $S^{3}$ [Rub95, Tho94], or whether a given polygonal knot in $\mathbb{R}^{3}$ is trivial [Hak61]. Indeed, both problems have recently been shown to lie in NP [Iva08, Sch04, HLP99]. The knot equivalence problem is also algorithmically decidable [Hak61, Hem79. Mat97], but nothing seems to be known about its complexity status. We refer the reader to the above-mentioned sources and to [AHT06] for further results, background and references.

In higher dimensions, all of these problems are undecidable. Markov [Mar58] showed that the homeomorphism problem for $d$-manifolds is algorithmically undecidable for every $d \geq 4$. For $d \geq 5$, this was strengthened by Novikov to the undecidability of recognizing $S^{d}$ (or any other fixed $d$-manifold), as was mentioned above. Nabutovsky and Weinberger [NW96] showed that for $d \geq 5$, it is algorithmically undecidable whether a given PL embedding $f: S^{d-2} \rightarrow \mathbb{R}^{d}$ is equivalent to the standard embedding (placing $S^{d-2}$ as the "equator" of the unit sphere $S^{d-1}$, say). For further undecidability results, see, e.g., [NW99] and the survey by Soare [Soa04].

Another direction of algorithmic research in topology is the computability of homotopy groups. While the fundamental group $\pi_{1}(X)$ is well-known to be uncomputable [Mar58], all higher homotopy groups of a given finite simply connected simplicial (or CW) complex are computable (Brown [Bro57]). There is also a \#P-hardness result of Anick [Ani89] for the computation of higher homotopy, but it involves CW complexes presented in a highly compact manner, and thus it does not seem to have any direct consequences for simplicial complexes. More recently, there appeared several works (Schön [Sch91], Smith [Smi98], and Rubio, Sergeraert, Dousson, and Romero, e.g. [RRS06]) aiming at making methods of algebraic topology, such as spectral sequences, "constructive"; the last of these has also resulted in an impressive software called KENZO.

A different line of research relevant to the embedding problem concerns linkless embeddings of graphs. Most notably, results of Robertson, Seymour, and Thomas [RST95] on linkless embeddings provide an interesting sufficient condition for embeddability of a 2-dimensional complex in $\mathbb{R}^{3}$, and they can thus be regarded as one of the few known positive results concerning $\mathrm{EMBED}_{2 \rightarrow 3}$. We will briefly discuss this in Section 6 .

\section{Preliminaries on PL topology}

Here we review definitions and facts related to piecewise linear (PL) embeddings. We begin with very standard things but later on we discuss notions and results which we found quite subtle (although they might be standard for specialists), in an area where it is sometimes tempting to consider as "obvious" something that is unknown or even false. Some more examples and open problems, which are not strictly necessary for the purposes of the present paper, but which helped us to appreciate some of the subtleties of the embeddability problem, are mentioned in Appendix C. For more information on PL topology, and for facts mentioned below without proofs, we refer to Rourke and Sanderson [RS82], Bryant [Bry02], or Buoncristiano [Buo03]. 
Simplicial complexes. We formally regard a simplicial complex as a geometric object, i.e., a collection $K$ (finite in our case) of closed simplices in some Euclidean space $\mathbb{R}^{n}$ such that if $\sigma \in K$ and $\sigma^{\prime}$ is a face of $\sigma$, then $\sigma^{\prime} \in K$ as well, and if $\sigma, \tau \in K$, then $\sigma \cap \tau \in K$ too. We write $V(K)$ for the vertex set, i.e., the set of all 0-dimensional simplices of $K$, and $|K|$ for the polyhedron of $K$, i.e., the union of all simplices in $K$. Often we do not strictly distinguish between a simplicial complex and its polyhedron; for example, by an embedding of $K$ in $\mathbb{R}^{d}$ we really mean an embedding of $|K|$ into $\mathbb{R}^{d}$.

The $k$-skeleton of $K$ consists of all simplices of $K$ of dimension at most $K$. A subcomplex of $K$ is a subset $L \subseteq K$ that is a simplicial complex. A simplicial complex $K^{\prime}$ is a subdivision of $K$ if $\left|K^{\prime}\right|=|K|$ and each simplex of $K^{\prime}$ is contained in some simplex of $K$.

Two simplicial complexes $K$ and $L$ are isomorphic if there is a face-preserving bijection $\varphi: V(K) \rightarrow V(L)$ of the vertex sets (that is, $F \subseteq V(K)$ is the vertex set of a simplex of $K$ iff $\varphi(F)$ is the vertex set of a simplex of $L)$. Isomorphic complexes have homeomorphic polyhedra. Up to isomorphism, a simplicial complex $K$ can be described purely combinatorially, by specifying which subsets of $V(K)$ form vertex sets of simplices of $K$.

We assume that the input to the embeddability problem is given in this form, i.e., as an abstract finite set system.

Linear and PL mappings of simplicial complexes. A linear mapping of a simplicial complex $K$ into $\mathbb{R}^{d}$ is a mapping $f:|K| \rightarrow \mathbb{R}^{d}$ that is linear on each simplex. More explicitly, each point $x \in|K|$ is a convex combination $t_{0} v_{0}+t_{1} v_{1}+\cdots+t_{s} v_{s}$, where $\left\{v_{0}, v_{1}, \ldots, v_{s}\right\}$ is the vertex set of some simplex $\sigma \in K$ and $t_{0}, \ldots, t_{s}$ are nonnegative reals adding up to 1 . Then we have $f(x)=t_{0} f\left(v_{0}\right)+t_{1} f\left(v_{1}\right)+\cdots+t_{s} f\left(v_{s}\right)$.

A PL mapping of $K$ into $\mathbb{R}^{d}$ is a linear mapping of some subdivision $K^{\prime}$ of $K$ into $\mathbb{R}^{d}$.

Embeddings. A general topological embedding of $K$ into $\mathbb{R}^{d}$ is any continuous mapping $f:|K| \rightarrow \mathbb{R}^{d}$ that is a homeomorphism of $|K|$ with $f(|K|)$. Since we only consider finite simplicial complexes, this is equivalent to requiring that $f$ be injective.

By contrast, for a PL embedding we require additionally that $f$ be PL, and for a linear embedding we are even more restrictive and insist that $f$ be (simplexwise) linear.

PL embeddings versus linear embeddings. In contrast to planarity of graphs, linear and PL embeddability do not always coincide in higher dimensions. Brehm [Bre83] constructed a triangulation of the Möbius strip that does not admit a linear embedding into $\mathbb{R}^{3}$. Using methods from the theory of oriented matroids, Bokowski and Guedes de Oliveira [BGdO00] showed that for any $g \geq 6$, there is a triangulation of the orientable surface of genus $g$ that does not admit a linear embedding into $\mathbb{R}^{3}$. In higher dimensions, Brehm and Sarkaria [BS92] showed that for every $k \geq 2$, and every $d$ with $k+1 \leq d \leq 2 k$, there is a $k$-dimensional simplicial complex $K$ that PL embeds into $\mathbb{R}^{d}$ but does not admit a linear embedding. Moreover, for any given $r \geq 0$, there is such a $K$ such that even the $r$-fold barycentric subdivision $K^{(r)}$ is not linearly embeddable into $\mathbb{R}^{d}$. Our Corollary 1.2 is another result of this kind.

On the algorithmic side, the problem of linear embeddability of a given finite simplicial complex into $\mathbb{R}^{d}$ is at least algorithmically decidable, and for $k$ and $d$ fixed, it even belongs to PSPACE (since the problem can easily be formulated as the solvability over 
the reals of a system of polynomial inequalities with integer coefficients, which lies in PSPACE [Ren92]).

PL structures. Two simplicial complexes $K$ and $L$ are $P L$ homeomorphic if there are a subdivision $K^{\prime}$ of $K$ and a subdivision $L^{\prime}$ of $L$ such that $K^{\prime}$ and $L^{\prime}$ are isomorphic.

Let $\Delta^{d}$ denote the simplicial complex consisting of all faces of a $d$-dimensional simplex (including the simplex itself), and let $\partial \Delta^{d}$ consist of all faces of $\Delta^{d}$ of dimension at most $d-1$. Thus, $\left|\Delta^{d}\right|$ is topologically $B^{d}$, the $d$-dimensional ball, and $\left|\partial \Delta^{d}\right|$ is topologically $S^{d-1}$.

A $d$-dimensional $P L$ ball is a simplicial complex PL homeomorphic to $\Delta^{d}$, and a $d$-dimensional $P L$ sphere is a simplicial complex PL homeomorphic to $\partial \Delta^{d+1}$. Let us mention that a (finite) simplicial complex $K$ is PL embeddable in $\mathbb{R}^{d}$ iff it is PL homeomorphic to a subcomplex of a $d$-dimensional PL ball (and similarly, $K$ is PL embeddable in $\left|\partial \Delta^{d+1}\right|$ iff it is PL homeomorphic to a subcomplex of a $d$-dimensional PL sphere).

One of the great surprises in higher-dimensional topology was the discovery that simplicial complexes with homeomorphic polyhedra need not be PL homeomorphic (the failure of the "Hauptvermutung"). In particular, there exist non-PL spheres, i.e., simplicial complexes homeomorphic to a sphere that fail to be PL spheres. More precisely, every simplicial complex homeomorphic to $S^{1}, S^{2}, S^{3}$, and $S^{4}$ is a PL sphere ${ }^{3}$ but there are examples of non-PL spheres of dimensions 5 and higher (e.g., the double suspension of the Poincaré homology 3-sphere).

A weak PL Schoenflies theorem. The well-known Jordan curve theorem states that if $S^{1}$ is embedded (topologically) in $\mathbb{R}^{2}$, the complement of the image has exactly two components. Equivalently, but slightly more conveniently, if $S^{1}$ is embedded in $S^{2}$, the complement has two components. The Schoenflies theorem asserts that in the latter setting, the closure of each of the components is homeomorphic to the disk $B^{2}$.

While the Jordan curve theorem generalizes to an arbitrary dimension (if $S^{d-1}$ is topologically embedded in $S^{d}$, the complement has exactly two components), the Schoenflies theorem does not. There are embeddings $h: S^{2} \rightarrow S^{3}$ such that the closure of one of the components of $S^{3} \backslash h\left(S^{2}\right)$ is not a ball; a well known example is the Alexander horned sphere.

The Alexander horned sphere is an infinitary construction; one needs to grow infinitely many "horns" from the embedded $S^{2}$ to make the example work. In higher dimensions, there are strictly finite examples, e.g., a 5-dimensional subcomplex $K$ of a 6dimensional PL sphere $S$ such that $|K|$ is topologically an $S^{5}$ (and $K$ is a non-PL sphere), but the closure of a component of $|S| \backslash|K|$ is not a topological ball (see Curtis and Zeeman [CZ61]).

Thus, one needs to put some additional conditions on the embedding to make a "higher-dimensional Schoenflies theorem" work. We will need the following version, in which we assume a $(d-1)$-dimensional PL sphere sitting in a $d$-dimensional PL sphere.

\footnotetext{
3 The proof for $S^{4}$ relies on the recent solution of the Poincare conjecture by Perelman.
} 
Theorem 2.1 (Weak PL Schoenflies Theorem). Let $f$ be a PL embedding of $\partial \Delta^{d}$ into $\partial \Delta^{d+1}$. Then the complement $\left|\partial \Delta^{d+1}\right| \backslash f\left(\left|\partial \Delta^{d}\right|\right)$ has two components, whose closures are topological d-balls.

For a proof of this theorem, see, e.g., [New60] or [Gla71]. A simple, inductive proof is to appear in the upcoming revised edition of the book [Buo03] by Buoncristiano and Rourke.

Let us remark that a "strong" PL Schoenflies theorem would claim that under the conditions of Theorem 2.1, the closure of each of the components is a PL ball, but the validity of this stronger statement is known only for $d \leq 3$, while for each $d \geq 4$ it is (to our knowledge) an open problem.

Genericity. First let us consider a linear mapping $f$ of a simplicial complex $K$ into $\mathbb{R}^{d}$. We say that $f$ is generic if $f(V(K))$ is a set of distinct points in $\mathbb{R}^{d}$ in general position.

If $\sigma, \tau \in K$ are disjoint simplices, then the intersection $f(\sigma) \cap f(\tau)$ is empty for $\operatorname{dim} \sigma+\operatorname{dim} \tau<d$ and it has at most one point for $\operatorname{dim} \sigma+\operatorname{dim} \tau=d$.

A PL mapping of $K$ into $\mathbb{R}^{d}$ is generic if the corresponding linear mapping of the subdivision $K^{\prime}$ of $K$ is generic.

A PL embedding can always be made generic (by an arbitrarily small perturbation).

Linking and linking numbers. Let $k, \ell$ be integers, and let $f: S^{k} \rightarrow \mathbb{R}^{k+\ell+1}$ and $g: S^{\ell} \rightarrow \mathbb{R}^{k+\ell+1}$ be PL embeddings with $f\left(S^{k}\right) \cap g\left(S^{\ell}\right)=\emptyset$ (so here we regard $S^{k}$ and $S^{\ell}$ as PL spheres). We will need two notions capturing how the images of $f$ and $g$ are "linked" (the basic example is $k=\ell=1$, where we deal with two disjoint simple closed curves in $\mathbb{R}^{3}$ ). For our purposes, we may assume that $f$ and $g$ are mutually generic (i.e. $f \sqcup g$, regarded as a PL embedding of the disjoint union $S^{k} \sqcup S^{\ell}$ into $\mathbb{R}^{k+\ell+1}$, is generic).

The images $f\left(S^{k}\right)$ and $g\left(S^{\ell}\right)$ are unlinked if $f$ can be extended to a PL mapping $\bar{f}: B^{k+1} \rightarrow \mathbb{R}^{k+\ell+1}$ of the $(k+1)$-dimensional ball such that $\bar{f}\left(B^{k+1}\right) \cap g\left(S^{\ell}\right)=\emptyset$.

To define the modulo 2 linking number of $f\left(S^{k}\right)$ and $g\left(S^{\ell}\right)$, we again extend $f$ to a PL mapping $\bar{f}: B^{k+1} \rightarrow \mathbb{R}^{k+\ell+1}$ so that $\bar{f}$ and $g$ are still mutually generic (but otherwise arbitrary). Then the modulo 2 linking number is the number of intersections between $\bar{f}\left(B^{k+1}\right)$ and $g\left(S^{\ell}\right)$ modulo 2 (it turns out that it does not depend on the choice of $\bar{f}$ ). In the following, we will use the phrase "odd linking number" instead of the more cumbersome "nonzero linking number modulo 2" (although "linking number" itself has not been properly defined).

These geometric definitions are quite intuitive. However, alternative (equivalent in our setting but more generally applicable) definitions are often used, phrased in terms of homology or mapping degree, which are in some respects easier to work with (e.g., they show that linking is symmetric, i.e., $f\left(S^{k}\right)$ and $g\left(S^{\ell}\right)$ are unlinked iff $g\left(S^{\ell}\right)$ and $f\left(S^{k}\right)$ are unlinked).

\section{Undecidability: Proof of Theorem 1.1}

We begin with a statement of Novikov's result mentioned in the introduction (undecidability of $S^{d}$ recognition for $d \geq 5$ ) in a form convenient for our purposes. 
Theorem 3.1 (Novikov). Fix $d \geq 5$. There is an effectively constructible sequence of simplicial complexes $\Sigma_{i}, i \in \mathbb{N}$, with the following properties:

(1) Each $\left|\Sigma_{i}\right|$ is a homology d-sphere.

(2) For each $i$, either $\Sigma_{i}$ is a PL $d$-sphere, or the fundamental group of $\Sigma_{i}$ is nontrivial (in particular, $\Sigma_{i}$ is not homeomorphic to the $d$-sphere).

(3) There is no algorithm that decides for every given $\Sigma_{i}$ which of the two cases holds.

We refer to the appendix in [Nab95] for a detailed proof.

We begin the proof of Theorem 1.1 with the following simple lemma.

Lemma 3.2. Let $\Sigma$ be a simplicial complex whose polyhedron is a homology $d$-sphere, $d \geq 2$. (The same proof works for any homology $d$-manifold.) Let $K$ be the $(d-1)$ skeleton of $\Sigma$. For every $d$-simplex $\sigma \in \Sigma$, the set $|K| \backslash \partial \sigma$ is path-connected (here $\partial \sigma$ is the relative boundary of $\sigma$ ).

Proof. By Lefschetz duality (see, e.g., [Mun84, Theorem 70.2]), $|\Sigma| \backslash \sigma$ is path connected. Indeed, Lefschetz duality yields $H^{0}(|\Sigma| \backslash \sigma) \cong H_{d}(\Sigma, \sigma)$ (homology with $\mathbb{Z}_{2}$ coefficients, say). The exact homology sequence of the pair $(\Sigma, \sigma)$, together with the fact that $\sigma$ is contractible, yields $H_{d}(\Sigma) \cong H_{d}(\Sigma, \sigma) \cong \mathbb{Z}_{2}$.

Next, we claim that if $\gamma$ is a path in $|\Sigma| \backslash \sigma$ connecting two points $x, y \in|K|$, then $x$ and $y$ can also be connected by a path in $|K| \backslash \partial \sigma$. Indeed, given a $d$-dimensional simplex $\tau \in \Sigma \backslash \sigma$, we have $\partial \tau \backslash \sigma$ path-connected. Hence we can modify $\gamma$ as follows: Letting $a:=\min \{t: \gamma(t) \in \tau\}$ and $b:=\max \{t: \gamma(t) \in \tau\}$, we replace the segment of $\gamma$ between $\gamma(a)$ and $\gamma(b)$ by a path $\eta$ in $\partial \tau \backslash \sigma$. Having performed this modification for every $\tau \in \Sigma \backslash \sigma$ (in some arbitrary order), we end up with a path connecting $x$ and $y$ that lies entirely within $|K| \backslash \partial \sigma$.

Lemma 3.3. Let $d \geq 2$. Suppose that $\Sigma$ is a homology $d$-sphere, and let $K$ be its $(d-1)$ skeleton.

(i) If $\Sigma$ is a PL sphere, then $K$ PL embeds into $\mathbb{R}^{d}$.

(ii) If $K$ PL embeds into $\mathbb{R}^{d}$, then $\Sigma$ is homeomorphic to $S^{d}$.

Proof. Part (i) is clear.

For part (ii), let us suppose that $f$ is a PL embedding of $K$ into $\mathbb{R}^{d}$. Since $K$ is compact, the image of $f$ is contained in some big $d$-dimensional simplex, and by taking this simplex as one facet of $\Delta^{d+1}$, we can consider $f$ as a PL embedding of $K$ into $\partial \Delta^{d+1}$. Consider a $d$-simplex $\sigma$ of $\Sigma$. By the weak PL Schoenflies theorem (Theorem 2.1,$\left|\partial \Delta^{d+1}\right| \backslash f(\partial \sigma)$ has two components, whose closures are topological $d$-balls. Moreover, since $|K| \backslash \partial \sigma$ is path-connected, its image under $f$ must be entirely contained in one of these components.

Therefore, we can use the closure of the other component to extend $f$ to a topological embedding of $\sigma$. By applying this reasoning to each $d$-face, we obtain a topological embedding $g$ of $\Sigma$ into $\partial \Delta^{d+1}$. It follows for instance from Alexander duality (see, e.g., [Mun84, Theorem 74.1]) that $g$ must be surjective, i.e., a homeomorphism. 
Proof of Theorem 1.1. The undecidability of $\operatorname{EMBED}_{(d-1) \rightarrow d}$ for $d \geq 5$ is an immediate consequence of Theorem 3.1 and Lemma 3.3

Proof of Corollary 1.2 Let us suppose that there is a recursive function $f$ contradicting the statement. That is, every $(d-1)$-dimensional $K$ with $n$ simplices that PL embeds in $\mathbb{R}^{d}$ has a subdivision with at most $f(n)$ simplices that embeds linearly. Then, given a $(d-1)$-dimensional complex $K$ with $n$ simplices, we could generate all subdivisions $K^{\prime}$ of $K$ with at most $f(n)$ simplices (see Acquistapace et al. [ABB90, Proposition 2.15]) and, using the PSPACE algorithms mentioned in Section 2, test the linear embeddability of each $K^{\prime}$ in $\mathbb{R}^{d}$. This would yield a decision algorithm for $\operatorname{EMBED}_{(d-1) \rightarrow d}$, contradicting Theorem 1.1 .

\section{Hardness of embedding 2-dimensional complexes in $\mathbb{R}^{4}$}

We will reduce the problem 3-SAT to $\mathrm{EMBED}_{2 \rightarrow 4}$. Given a 3-CNF formula $\varphi$, we construct a 2-dimensional simplicial complex $K$ that is PL embeddable in $\mathbb{R}^{4}$ exactly if $\varphi$ is satisfiable.

First we define two particular 2-dimensional simplicial complexes $G$ (the clause gadget) and $X$ (the conflict gadget). They are closely related to the main example of Freedman et al. [FKT94]: $X$ is taken over exactly, and $G$ is a variation on a construction in [FKT94] (which, in turn, is similar in some respects to an example of Segal and Spież [SS92], with some of the ideas going back to Van Kampen [vK32]).

\subsection{The clause gadget}

To construct $G$, we begin with a 6-dimensional simplex on the vertex set $\left\{v_{0}, v_{1}, \ldots, v_{6}\right\}$, and we let $F$ be the 2 -skeleton of this simplex ( $F$ for "full" skeleton). Then we make a hole in the interior of the three triangles (2-simplices) $v_{0} v_{1} v_{2}, v_{0} v_{1} v_{3}$, and $v_{0} v_{2} v_{3}$. That is, we subdivide each of the triangles and from each of these subdivisions we remove a small triangle in the middle, as indicated in Fig. $\left.1\right|^{4}$ This yields the simplicial complex $G$.

Let $\omega_{1}, \omega_{2}, \omega_{3}$ be the three small triangles we have removed (where $\omega_{1}$ comes from the triangle $v_{0} v_{2} v_{3}$ etc.). We call them the openings of $G$ and we let $O_{G}:=\left\{\omega_{1}, \omega_{2}, \omega_{3}\right\}$ be the set of openings. Thus, $G \cup O_{G}$ is a subdivision of the full 2-skeleton $F$.

If we remove from $F$ the vertices $v_{0}, v_{1}, v_{2}$ and all simplices containing them, we obtain the boundary of the 3 -simplex $\left\{v_{3}, v_{4}, v_{5}, v_{6}\right\}$. Topologically it is an $S^{2}$, we call it the complementary sphere of the opening $\omega_{3}$, and we denote it by $S_{\omega_{3}}$. The complementary spheres of the openings $\omega_{1}$ and $\omega_{2}$ are defined analogously. The following lemma is a variation on results in Van Kampen [vK32]:

\footnotetext{
4 Alternatively, we could also make the clause gadget by simply removing the triangles $v_{0} v_{1} v_{2}$, $v_{0} v_{1} v_{3}$, and $v_{0} v_{2} v_{3}$ from $F$. However, the embedding of the resulting complex $K$ for satisfiable formulas $\varphi$ would become somewhat more complicated.
} 


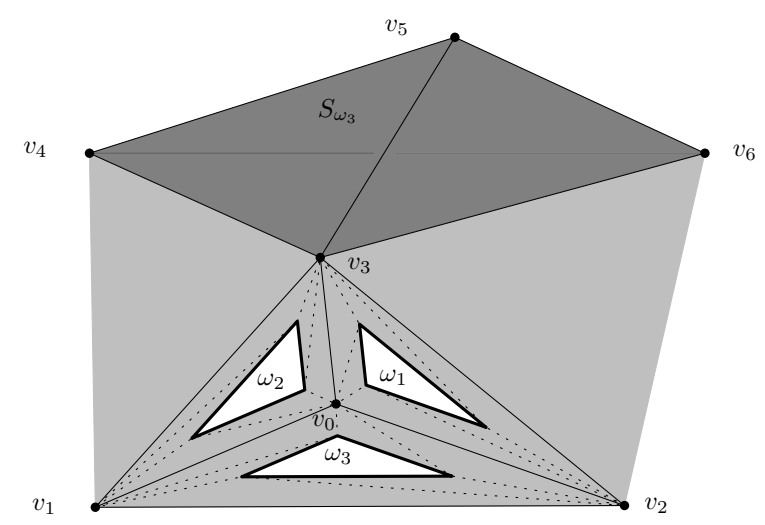

Fig. 1. The clause gadget $G$, its openings, and one of the complementary spheres.

\section{Lemma 4.1.}

(i) For every generic PL embedding $f$ of $G$ into $\mathbb{R}^{4}$ there is at least one opening $\omega \in O_{G}$ such that the images of the boundary $\partial \omega$ and of the complementary sphere $S_{\omega}$ have odd linking number.

(ii) For every opening $\omega \in O_{G}$ there exists an embedding of $G$ into $\mathbb{R}^{4}$ in which only $\partial \omega$ is linked with its complementary sphere. More precisely, there exists a generic linear mapping of the full 2-skeleton $F$ into $\mathbb{R}^{4}$ whose restriction to $\left|G \cup O_{G} \backslash\{\omega\}\right|$ is an embedding.

Proof. (i) This is very similar to Lemma 6 in [FKT94]. Let $f_{0}$ be a generic PL map (not necessarily an embedding) of $F$ into $\mathbb{R}^{4}$. Van Kampen proved that

$$
\sum_{\{\sigma, \tau\}}\left|f_{0}(\sigma) \cdot f_{0}(\tau)\right|
$$

is always odd, where $\left|f_{0}(\sigma) \cdot f_{0}(\tau)\right|$ denotes the number of intersections between the image of $\sigma$ and the image of $\tau$, and the sum is over all unordered pairs of disjoint 2dimensional simplices $\sigma, \tau \in F$ (the genericity of $f_{0}$ guarantees that the intersection $f_{0}(\sigma) \cap f_{0}(\tau)$ consists of finitely many points). (See Appendix $\mathrm{D}$ for a wider context of this result.)

Now let us consider a generic PL embedding $f$ of $G$ into $\mathbb{R}^{4}$, and let us extend it piecewise linearly and generically (and otherwise arbitrarily) to the openings of $G$. The resulting map can also be regarded as a generic PL map $f_{0}$ of $F$ into $\mathbb{R}^{4}$. For such an $f_{0}$, $\left|f_{0}(\sigma) \cdot f_{0}(\tau)\right|$ can be nonzero only if $\sigma$ contains an opening $\omega$ of $G$ and $\tau$ belongs to its complementary sphere $S_{\omega}$ (or the same situation with $\sigma$ and $\tau$ interchanged). Thus, for at least one $\omega \in O_{G}, f_{0}(\omega)$ intersects $f\left(S_{\omega}\right)$ in an odd number of points, and this means exactly that $f(\partial \omega)$ and $f\left(S_{\omega}\right)$ have odd linking number.

(ii) It suffices to exhibit a generic linear map $f_{0}$ of $F$ into $\mathbb{R}^{4}$ such that the images of two disjoint 2-simplices intersect (in a single point), and this intersection is the only multiple point of $f_{0}$. Such a mapping was constructed by Van Kampen [vK32]: five of the 
vertices are placed as vertices of a 4-dimensional simplex in $\mathbb{R}^{4}$, and the remaining two are mapped into the interior of that simplex.

\subsection{The conflict gadget}

To construct $X$, we start with the 1-dimensional simplicial complex $E$ shown in Fig. 2 (left), consisting of two triangular loops $\Sigma_{a}$ and $\Sigma_{b}$ and an edge $c$ connecting them. We also fix an orientation of $\Sigma_{a}, \Sigma_{b}$, and $c$ (marked by arrows). Then we take a disk $D$ and we attach its boundary to $E$ as indicated in Fig. 2 (right); the disk is triangulated sufficiently finely so that the result of the attachment is still a simplicial complex. This is the complex $X$.
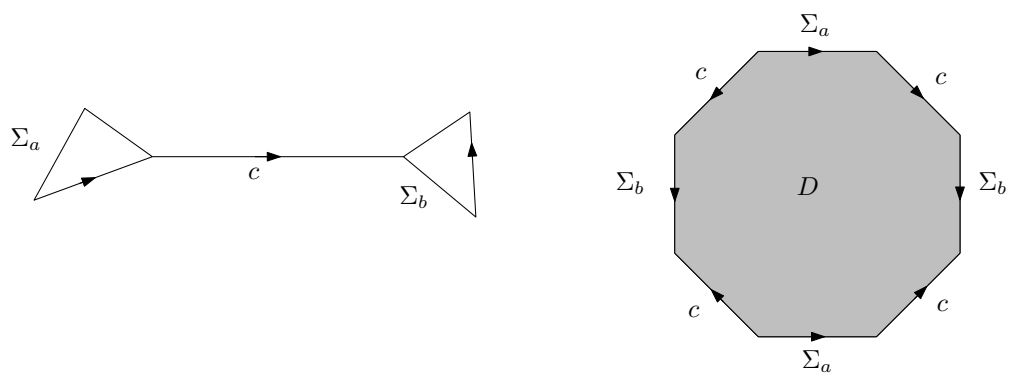

Fig. 2. Attaching a disk to the polygonal line $E$.

We observe that topologically, $X$ is a "squeezed torus" (the reader may want to recall the usual construction of a torus by gluing the opposite sides of a square; this well-known construction would be obtained from the attachment as above if the edge $c$ were contracted to a point). Fig. 3 shows such a squeezed torus embedded in $\mathbb{R}^{3}$ (with the loops $\Sigma_{a}$ and $\Sigma_{b}$ drawn circular rather than triangular).

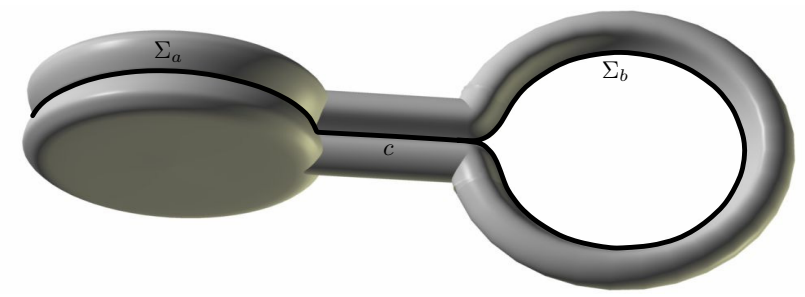

Fig. 3. A 3-dimensional embedding of the conflict gadget.

\section{Lemma 4.2.}

(i) [FKT94, Lemma 7] Let $S_{a}$ and $S_{b}$ be PL 2-spheres. Then there is no PL embedding $f$ of $S_{a} \sqcup S_{b} \sqcup X$ (disjoint union) into $\mathbb{R}^{4}$ such that

- the 1-sphere $f\left(\Sigma_{a}\right)$ and the 2-sphere $f\left(S_{a}\right)$ have odd linking number, and so do $f\left(\Sigma_{b}\right)$ and $f\left(S_{b}\right)$;

- $f\left(\Sigma_{a}\right)$ and $f\left(S_{b}\right)$ are unlinked, and so are $f\left(\Sigma_{b}\right)$ and $f\left(S_{a}\right)$. 
(ii) Let $f$ be a generic linear embedding of $E$ in $\mathbb{R}^{3}$ (not $\mathbb{R}^{4}$ this time) such that $f\left(\Sigma_{a}\right)$ and $f\left(\Sigma_{b}\right)$ are unlinked, and let $\delta>0$. Then there is a PL embedding $\bar{f}$ of $X$ in $\mathbb{R}^{3}$ extending $f$ whose image is contained in the set $N=N(f, \delta):=N\left(T_{a}, \delta\right) \cup$ $N\left(f\left(\Sigma_{b}\right), \delta\right) \cup N(f(c), \delta)$, where $T_{a}$ is the triangle bounded by the loop $f\left(\Sigma_{a}\right)$ and $N(A, \delta)$ denotes the $\delta$-neighborhood of a set $A$ (in $\mathbb{R}^{3}$ in our case) 5 (Symmetrically, and this is the main point of the construction, we can also embed $X$ into $N\left(f\left(\Sigma_{a}\right), \delta\right) \cup N\left(T_{b}, \delta\right) \cup N(f(c), \delta)$, thus leaving a hole on the other side.)

For a proof of part (i) we refer to [FKT94] (a few words about the basic approach of the proof will be said in the proof of Lemma 5.3 below), and for part (ii) to Fig. 3 .

\subsection{The reduction}

Let the given 3-CNF formula be $\varphi=C_{1} \wedge \cdots \wedge C_{m}$, where each $C_{i}$ is a clause with three literals (each literal is either a variable or its negation). For each $C_{i}$, we take a copy of the clause gadget $G$ and we denote it by $G_{i}$ (the $G_{i}$ have pairwise disjoint vertex sets). We fix a one-to-one correspondence between the literals of $C_{i}$ and the openings of $G_{i}$, letting $\omega(\lambda)$ be the opening corresponding to a literal $\lambda$.

Let us say that a literal $\lambda$ in a clause $C_{i}$ is in conflict with a literal $\mu$ in a clause $C_{j}$ if both $\lambda$ and $\mu$ involve the same variable $x$ but one of them is $x$ and the other the negation $\bar{x}$. For convenience we assume, without loss of generality, that two literals from the same clause are never in conflict.

Let $\Xi$ consist of all (unordered) pairs $\{\omega(\lambda), \omega(\mu)\}$ of openings corresponding to pairs $\{\lambda, \mu\}$ of conflicting literals in $\varphi$. For every pair $\{\omega, \psi\} \in \Xi$ we take a fresh copy $X_{\omega \psi}$ of the conflict gadget $X$. We identify the loop $\Sigma_{a}$ in $X_{\omega \psi}$ with the boundary $\partial \omega$ and the loop $\Sigma_{b}$ with $\partial \psi$ (the rest of $X_{\omega \psi}$ is disjoint from the clause gadgets and the other conflict gadgets).

The simplicial complex $K$ assigned to the formula $\varphi$ is

$$
K:=\left(\bigcup_{i=1}^{m} G_{i}\right) \cup\left(\bigcup_{\{\omega, \psi\} \in \Xi} X_{\omega \psi}\right) .
$$

It remains to show that $K$ is PL embeddable in $\mathbb{R}^{4}$ exactly if $\varphi$ is satisfiable.

Nonembeddability for unsatisfiable formulas. This is a straightforward consequence of Lemmas 4.1.i) and 4.2 (i).

Indeed, if $f$ is a PL embedding of $K$ into $\mathbb{R}^{4}$, which we may assume to be generic, there is an opening in each clause gadget $G_{i}$ such that $f\left(\partial \omega_{i}\right)$ has odd linking number with the complementary sphere $f\left(S_{\omega_{i}}\right)$; let us call it an occupied opening of $G_{i}$. Since $\varphi$ is not satisfiable, whenever we choose one literal from each clause, there are two of the chosen literals in conflict. Thus, there are two occupied openings $\omega \in O_{G_{i}}$ and $\psi \in O_{G_{j}}$ that are connected by a conflict gadget $X_{\omega \psi}$.

\footnotetext{
5 Formally $N(A, \delta)=\left\{x \in \mathbb{R}^{3}: \operatorname{dist}(x, A) \leq \delta\right\}$, where $\operatorname{dist}(x, A)$ is the Euclidean distance of $x$ from the set $A$.
} 
Then the supposed PL embedding $f$ provides us an embedding as in Lemma 4.2 i) with $S_{a}=S_{\omega}, S_{b}=S_{\psi}$, and $X=X_{\omega \psi}$. Concerning the assumptions in the lemma, we already know that $f\left(S_{\omega}\right)$ and $f(\partial \omega)$ have odd linking number, and so do $f\left(S_{\psi}\right)$ and $f(\partial \psi)$. It remains to observe that $f(\partial \omega)$ cannot be linked with $f\left(S_{\psi}\right)$ (and vice versa), since $G_{i}$ contains a disk bounded by $\partial \omega$ : for example (refer to Fig. 1 ,,$\partial \omega_{3}$ is the boundary of the disk consisting of the triangles $v_{0} v_{1} v_{4}, v_{0} v_{2} v_{4}, v_{1} v_{2} v_{4}$ and the triangles in the subdivision of $v_{0} v_{1} v_{2}$ different from $\omega_{3}$. So the lemma applies and $K$ is not embeddable.

Embedding for satisfiable formulas. Given a satisfying assignment for $\varphi$, we choose a witness literal $\lambda_{i}$ for each clause $C_{i}$ that is true under the given assignment (and we will refer to the remaining two literals of $C_{i}$ as non-witness ones). No two witness literals can be in conflict.

We describe an embedding of $K$ into $\mathbb{R}^{4}$ corresponding to this choice of witness literals.

Let us choose distinct points $p_{1}, \ldots, p_{m} \in \mathbb{R}^{4}$. For each $i=1, \ldots, m$, we let $f_{i}$ be a generic linear embedding of the clause gadget $G_{i}$ into a small neighborhood of $p_{i}$ (and far from the other $p_{j}$ ) as in Lemma 4.1 (ii), where the role of $\omega$ in the lemma is played by the witness opening of $G_{i}$ (i.e., the one corresponding to the witness literal of $C_{i}$ ). In particular, the interiors of the triangles bounded by $f_{i}\left(\partial \omega^{\prime}\right)$ and by $f_{i}\left(\partial \omega^{\prime \prime}\right)$ are disjoint from $f_{i}\left(G_{i}\right)$, where $\omega^{\prime}$ and $\omega^{\prime \prime}$ are the non-witness openings of $G_{i}$.

Taking all the $f_{i}$ together defines an embedding $f$ of the union of the clause gadgets, and it remains to embed the conflict gadgets.

To this end, we will assign to each conflict gadget $X_{\omega \psi}$ a "private" set $P_{\omega \psi} \subset \mathbb{R}^{4}$ homeomorphic to the 3-dimensional set $N$ from Lemma 4.2 (ii), and we will embed $X_{\omega \psi}$ into $P_{\omega \psi}$. Each $P_{\omega \psi}$ will be disjoint from all other $P_{\omega^{\prime}} \psi^{\prime}$ and also from all the images $f\left(G_{i}\right)$, except that $P_{\omega \psi}$ has to contain the loops $f(\partial \omega)$ and $f(\partial \psi)$ where the conflict gadget $X_{\omega \psi}$ should be attached. In order to fit enough almost-disjoint homeomorphic copies of $N$ into the space, we will "fold" them suitably.

We know that for every pair $\{\omega, \psi\}$ of openings connected by a conflict gadget, at least one of $\omega$ and $\psi$ is non-witness. Let us choose the notation so that $\omega$ is non-witness and thus unoccupied in the embedding $f$.

We will build $P_{\omega \psi}$ from three pieces: a set $Q_{\omega \psi}^{+}$that plays the role of $N\left(T_{a}, \delta\right)$ in Lemma 4.2 (ii), a set $Q_{\psi \omega}$ that plays the role of $N\left(f\left(\Sigma_{b}\right), \delta\right)$, and a "connecting ribbon" in the role of $N(f(c), \delta)$.

Now let $\omega$ be an opening of some $G_{i}$, witness or non-witness. Let $t$ be the number of openings $\psi$ that are connected to $\omega$ by a conflict gadget. The sets $Q_{\omega \psi}$ and $Q_{\omega \psi}^{+}$we want to construct are indexed by these $\psi$, but with some abuse of notation, we will now regard them as indexed by an index $j$ running from 1 to $t$, i.e., as $Q_{\omega 1}$ through $Q_{\omega t}$ (and similarly for $Q_{\omega \psi}^{+}$).

For concise notation let us write $\Sigma=f(\partial \omega)$ and let $T$ be the triangle in $\mathbb{R}^{4}$ having $\Sigma$ as the boundary. Let $\varepsilon>0$ be a parameter and let $T^{\varepsilon}:=\{x \in T: \operatorname{dist}(x, \partial T) \leq \varepsilon\}$ be the part of $T$ at most $\varepsilon$ away from the boundary of $T$. Since the subdivided triangle in $G_{i}$ containing $\omega$ in its interior is embedded linearly by $f$, there is an $\varepsilon>0$ such that if we start at a point $x \in T^{\varepsilon}$ and go a distance at most $\varepsilon$ in a direction orthogonal to $T$, we do 


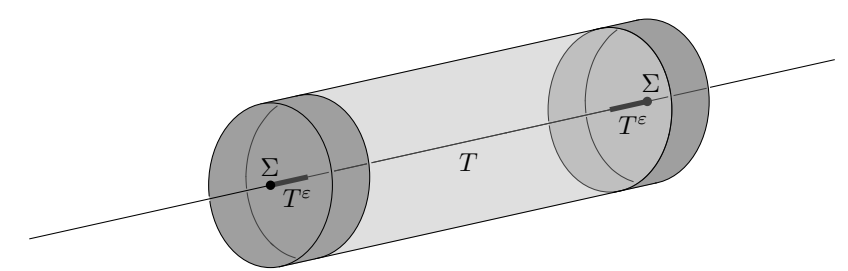

Fig. 4. A free region around the triangle $T$; illustration in $\mathbb{R}^{3}$ instead of $\mathbb{R}^{4}$.

not hit $f\left(G_{i}\right)$. Moreover, if $\omega$ is non-witness and thus all of $T$ is free of $f\left(G_{i}\right)$, we can take any $x \in T$ with the same result. Fig. 4 tries to illustrate this in dimension one lower, where we have a segment $T$ in $\mathbb{R}^{3}$ instead of a triangle $T$ in $\mathbb{R}^{4}$. Thus, there are a set $Q_{\omega} \subset \mathbb{R}^{4}$ with $Q_{\omega} \cap f\left(G_{i}\right)=\Sigma$ and a homeomorphism (actually, a linear isomorphism) $h: Q_{\omega} \rightarrow T^{\varepsilon} \times B^{2}$ with $h\left(T^{\varepsilon}\right)=T^{\varepsilon} \times\{0\}$, where 0 is the center of the disk $B^{2}$. Similarly, if $\omega$ is non-witness, there are $Q_{\omega}^{+}$and $h^{+}: Q_{\omega}^{+} \rightarrow T \times B^{2}$ with $h^{+}(T)=T \times\{0\}$.

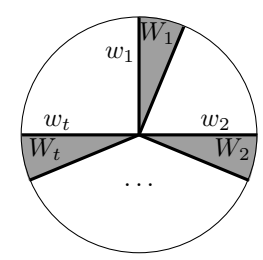

Fig. 5. The wedges.

Let $W_{1}, \ldots, W_{t} \subset B^{2}$ be disjoint wedges as in Fig. 5. and let $w_{j}$ consist of the two radii bounding $W_{j}$. We set

$$
Q_{\omega j}:=h^{-1}\left(\left(\Sigma \times W_{j}\right) \cup\left(T^{\varepsilon} \times w_{j}\right)\right), \quad Q_{\omega j}^{+}:=\left(h^{+}\right)^{-1}\left(\left(\Sigma \times W_{j}\right) \cup\left(T \times w_{j}\right)\right) .
$$

As Fig. 6 tries to illustrate, $Q_{\omega j}^{+}$is homeomorphic to a 3-dimensional neighborhood of $T$

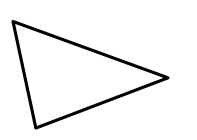

$(\Sigma$

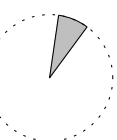

$\left.W_{j}\right)$

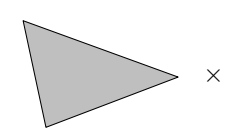

( $\quad \times$

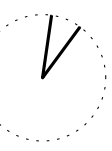

$\left.w_{j}\right)$
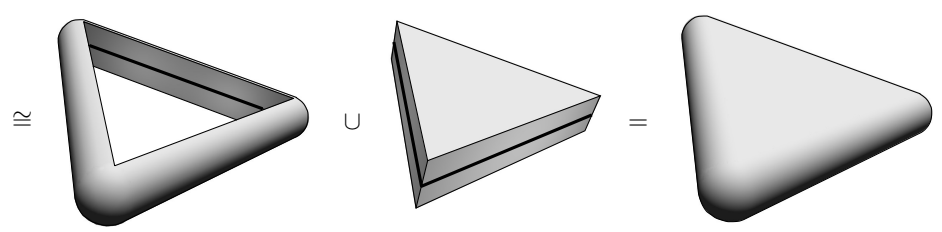

Fig. 6. Folding a 3-dimensional neighborhood in $\mathbb{R}^{4}$. 
(by a homeomorphism sending $T$ to $T$ ), and $Q_{\omega j}$ is similarly homeomorphic to a 3dimensional neighborhood of $\Sigma$. Thus, the sets $Q_{\omega j}$ and $Q_{\omega j}^{+}$can indeed play the roles of $N\left(f\left(\Sigma_{b}\right), \delta\right)$ and $N\left(T_{a}, \delta\right)$, respectively, in Lemma 4.1.ii).

It remains to construct the "connecting ribbons": for every conflict gadget $X_{\omega \psi}$, we want to connect a vertex of $f(\partial \omega)$ to a vertex of $f(\partial \psi)$ by a narrow 3-dimensional "ribbon" (it need not be straight since we are looking only for PL homeomorphic copies of $N$ ).

We observe that each of the sets $Q_{\omega j}$ and $Q_{\omega j}^{+}$can be deformation-retracted to the corresponding loop $f(\partial \omega)$ or to the corresponding triangle, respectively. It follows that the complement of the union $U$ of all the $Q_{\omega j}, Q_{\omega j}^{+}$, and $f\left(G_{i}\right)$ is path-connected (formally, this follows from Alexander duality, since this union is homotopy equivalent to a 2-dimensional space). Since all the embeddings considered are piecewise linear, any two points on the boundary of $U$ can be connected by a PL path within $\mathbb{R}^{4} \backslash U$.

Thus, the 3-dimensional "ribbon" connecting $f(\partial \omega)$ to $f(\partial \psi)$ can first go within the appropriate $Q_{\omega j}$ to a point on the boundary, then continue along a path connecting this boundary point to a boundary point of $Q_{\psi j^{\prime}}$, and then reach $f(\partial \psi)$ within $Q_{\psi j^{\prime}}$.

In this way, we have allocated the desired "private" sets $P_{\omega \psi}$ for all conflict gadgets $X_{\omega \psi}$, and hence $K$ can be PL embedded in $\mathbb{R}^{4}$ as claimed. This finishes the proof of the special case $k=2, d=4$ of Theorem 1.3 .

\section{NP-hardness for higher dimensions}

In this section we prove all the remaining cases of Theorem 1.3 . The proof is generally very similar to the case $k=2, d=4$ treated above: we will again reduce 3 -SAT using clause gadgets and conflict gadgets, but the construction of the gadgets and of their embeddings require additional work.

By the monotonicity of EMBED $k \rightarrow d$ in $k$ mentioned in Section 1, it suffices to consider $d \geq 5$ and $k=\lceil(2 d-2) / 3\rceil$. In the construction we will often use the integer $\ell:=d-k-1$.

\subsection{The clause gadget}

The clause gadget $G=G(k, \ell)$ is very similar to a construction of Segal and Spież [SS92]. We use the parameters $k, \ell, d$ as above. For the purposes of the present section we need that $1 \leq \ell<k$ and $d-\ell=k+1 \geq 3$ (which are easy to verify using the definitions of $k$ and $\ell$ and the assumption $d \geq 5$ ).

For the parameters $k, \ell, d$ as above, we first define a simplicial complex $F=F(k, \ell)$ on the vertex set $V:=\left\{v_{0}, v_{1}, \ldots, v_{d+1}, p\right\}$ as the union $F:=F_{0} \cup C_{p}$ of the following two sets of simplices:

- $F_{0}$ is the $k$-skeleton of the $(d+1)$-simplex with vertex set $\left\{v_{0}, \ldots, v_{d+1}\right\}$;

- $C_{p}$ consists of all the $(\ell+1)$-dimensional simplices on $V$ that contain $p$. 


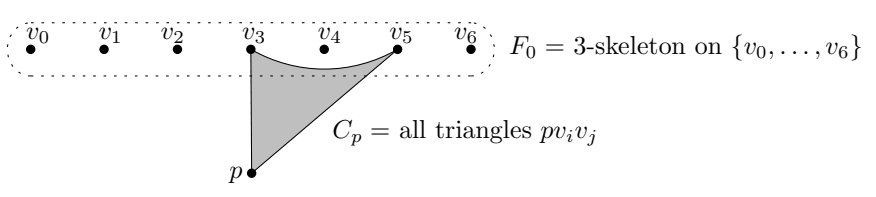

Fig. 7. A schematic illustration of $F(3,1)$.

See Fig. 7 for a schematic illustration; let us also note that for $d=4, k=2, \ell=1$ we would get exactly the $F$ as in Section 4.1

Let us consider some $\sigma \in C_{p}$. By removing from $F$ all simplices intersecting $\sigma$ (including $\sigma$ ), we obtain the $k$-skeleton of a $(k+1)$-simplex, i.e., an $S^{k}$, which we call the complementary sphere $S_{\sigma}$.

Next, we fix three $(\ell+1)$-dimensional simplices $\sigma_{1}, \sigma_{2}, \sigma_{3} \in C_{p}$, say $\sigma_{1}:=$ $p v_{0} v_{2} v_{3} \cdots v_{\ell+1}, \sigma_{2}:=p v_{0} v_{1} v_{3} \cdots v_{\ell+1}$, and $\sigma_{3}:=p v_{0} v_{1} v_{2} v_{4} \cdots v_{\ell+1}$. As in Section 4.1. we make a hole in the interior of each $\sigma_{i}$, i.e., we subdivide each $\sigma_{i}, i=1,2,3$, and we remove a small $(\ell+1)$-simplex $\omega_{i}$ in the middle. This yields the simplicial complex $G=G(k, \ell)$.

The $\omega_{i}$ are again called the openings of $G$, and we set $O_{G}:=\left\{\omega_{1}, \omega_{2}, \omega_{3}\right\}$. The complementary sphere $S_{\omega_{i}}$ is defined, with some abuse of notation, as the complementary sphere of the simplex $\sigma_{i} \in C_{p}$ that contains $\omega_{i}$.

Lemma 5.1 (Higher-dimensional version of Lemma 4.1).

(i) For every generic PL embedding $f$ of $G$ into $\mathbb{R}^{d}$ there is at least one opening $\omega \in O_{G}$ such that the images of the boundary $\partial \omega$ and of the complementary sphere $S_{\omega}$ have odd linking number.

(ii) For every opening $\omega \in O_{G}$ there exists a generic linear embedding of $G$ into $\mathbb{R}^{d}$ in which the boundaries of the two openings different from $\omega$ are unlinked with their complementary spheres.

Proof. Part (ii) is established in the proof of Lemma 1.1 in Segal and Spież [SS92] (generalizing Van Kampen's embedding mapping mentioned in the proof of Lemma 4.1(ii)). They construct a PL embedding of $F(k, \ell)$ (which they call $P(k, \ell)$, while their $n$ is our $d-1)$, but inspecting the first two paragraphs of their proof reveals that their embedding is actually linear (in the subsequent paragraphs, they modify the embedding on the interior of one of the $(\ell+1)$-simplices from $C_{p}$, but this serves only to show the claim about linking number).

For part (i), it clearly suffices to prove the following:

Claim. For any generic PL mapping $g$ of $F$ in $\mathbb{R}^{d}$ whose restriction to $F_{0}$ is an embedding, there is an $(\ell+1)$-dimensional simplex $\sigma \in C_{p}$ such that $\left|g(\sigma) \cap g\left(S_{\sigma}\right)\right|$ is odd.

This claim follows easily from the proof of Lemma 1.4 in Segal and Spież [SS92]. Indeed, they give a procedure that, given a generic PL map $g_{1}$ of $F$ into $\mathbb{R}^{d}$ such that $\left|g_{1}(\sigma) \cap g_{1}\left(S_{\sigma}\right)\right|$ is even for some $\sigma$, yields a new generic PL map $g_{2}$ with 
$g_{2}(\sigma) \cap g_{2}\left(S_{\sigma}\right)=\emptyset$ and such that there are no new intersections between images of disjoint simplices (compared to $\left.g_{1}\right) 6$

Assuming that there is a $g$ contradicting the claim, after finitely many applications of the procedure we arrive at a generic PL mapping $\tilde{g}$ such that $\tilde{g}(\sigma) \cap \tilde{g}\left(S_{\sigma}\right)=\emptyset$ for every $\sigma \in C_{p}$. We claim that then

$$
\tilde{g}(\tau) \cap \tilde{g}\left(\tau^{\prime}\right)=\emptyset \quad \text { for every } \tau, \tau^{\prime} \in F \text { with } \tau \cap \tau^{\prime}=\emptyset .
$$

Indeed, if 11 fails for some $\tau, \tau^{\prime}$, one of $\tau, \tau^{\prime}$ (say $\tau^{\prime}$ ) must belong to $C_{p}$, since $g$ restricted to $F_{0}$ is an embedding. But then we get $\tau \in S_{\tau^{\prime}}$-a contradiction. Hence (1) holds. But no generic PL mapping $\tilde{g}$ satisfying (1) exists according to [SS92] (end of the proof of Lemma 1.4). This proves the claim and thus also part (i) of Lemma 5.1 .

Let us remark that a perhaps more conceptual proof of part (i) can be obtained using the results of Shapiro [Sha57] on the "generalized Van Kampen obstruction", but we would need many preliminaries to present it.

\subsection{The conflict gadget}

Here we construct the conflict gadget $X=X(\ell)$, which depends only on the parameter $\ell$, and whose dimension is $2 \ell$. The conflict gadget $X$ in Section 4.2 is essentially the same as the following construction for $\ell=1$, up to minor formal differences. In addition to the inequalities among the parameters mentioned earlier, here we also need $2 \ell \leq k$ (which again holds in our setting).

In the $\ell=1$ case we attached a 2 -dimensional disk along its boundary to the 1-dimensional complex $E$. The $\ell$-dimensional version of $E$ consists of two disjoint copies $\Sigma_{a}^{\ell}$ and $\Sigma_{b}^{\ell}$ of the boundary of the $(\ell+1)$-simplex connected by an edge $c$ (see Fig. 8 . To this

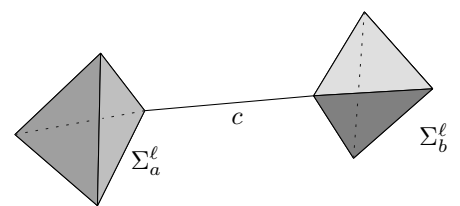

Fig. 8. A higher-dimensional version of $E$.

$E$ we are going to attach the $(2 \ell)$-dimensional ball $B^{2 \ell}$ along its boundary. For $\ell=1$ the result was topologically a "squeezed" version of the 2-dimensional torus $S^{1} \times S^{1}$; for larger $\ell$ it is going to be the higher-dimensional "torus" $S^{\ell} \times S^{\ell}$, again suitably squeezed.

Attaching a ball to $S^{\ell} \vee S^{\ell}$. Before defining $X$ itself, we define a certain mapping $g: S^{2 \ell-1} \rightarrow S^{\ell} \vee S^{\ell}$, where $S^{\ell} \vee S^{\ell}$ is a wedge of two spheres, to be defined below.

\footnotetext{
6 The procedure requires $d-\ell \geq 3$, which is satisfied in our case. In [SS92] this inequality is reversed by mistake.
} 
This construction is based on the Whitehead product in homotopy theory. As we will see, attaching the boundary of $B^{2 \ell}$ to $S^{\ell} \vee S^{\ell}$ via $g$ results topologically in $S^{\ell} \times S^{\ell}$ (without any squeezing).

The wedge $S^{\ell} \vee S^{\ell}$ consists of two copies of the sphere $S^{\ell}$ glued together at one point. For our purposes, we represent $S^{\ell} \vee S^{\ell}$ concretely as follows. We consider $S^{\ell}$ geometrically as the unit sphere in $\mathbb{R}^{\ell+1}$, we choose a distinguished point $s_{0}=(1,0, \ldots, 0) \in S^{\ell}$, and we let $S^{\ell} \vee S^{\ell}$ be the subspace $\left(S^{\ell} \times\left\{s_{0}\right\}\right) \cup\left(\left\{s_{0}\right\} \times S^{\ell}\right)$ of $\mathbb{R}^{\ell+1} \times \mathbb{R}^{\ell+1}=\mathbb{R}^{2 \ell+2}$. For $\ell=1$, we thus get two unit circles lying in perpendicular 2-flats in $\mathbb{R}^{4}$ and meeting at the point $\left(s_{0}, s_{0}\right)$.

To define the map $g$, we need to represent the ball $B^{2 \ell}$ not as the standard Euclidean unit ball, but rather as the product $B^{\ell} \times B^{\ell}$ (which is clearly homeomorphic to $B^{2 \ell}$ ). Then we have

$$
S^{2 \ell-1} \cong \partial\left(B^{\ell} \times B^{\ell}\right)=\left(B^{\ell} \times S^{\ell-1}\right) \cup\left(S^{\ell-1} \times B^{\ell}\right) ;
$$

see the left part of Fig. 9 for the (rather trivial) case $\ell=1$. (Indeed, for arbitrary sets $A \subseteq \mathbb{R}^{m}$ and $B \subseteq \mathbb{R}^{n}$ we have $\partial(A \times B)=(A \times \partial B) \cup(\partial A \times B)$, as is easy to check. $)$

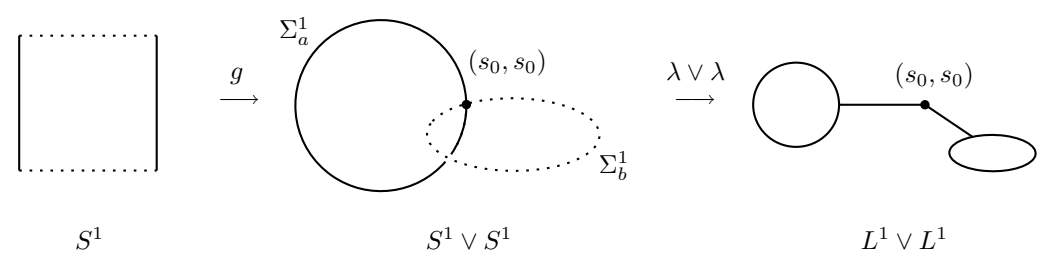

Fig. 9. Representing $S^{1}$ as $\left(B^{1} \times S^{0}\right) \cup\left(S^{0} \times B^{1}\right)$ (left); mapping it to $S^{1} \vee S^{1}$ (middle); squeezing the $S^{1}$ 's to "lollipops" (right). We note that $S^{1} \vee S^{1}$ and $L^{1} \vee L^{1}$ actually live in $\mathbb{R}^{4}$.

As is well known, if we shrink the boundary of an $n$-ball to a single point, the result is an $n$-sphere. Let us fix a mapping $\gamma: B^{\ell} \rightarrow S^{\ell}$ that sends all of $\partial B^{\ell}$ to the distinguished point $s_{0}$ and is a homeomorphism on the interior of $B^{\ell}$. Now we are ready to define the map $g$. Namely, we define $\bar{g}: B^{2 \ell} \rightarrow S^{\ell} \times S^{\ell}$ by

$$
\bar{g}(x, y)=(\gamma(x), \gamma(y)),
$$

where we still consider $B^{2 \ell}$ as $B^{\ell} \times B^{\ell}$ and $x$ comes from the first $B^{\ell}$ and $y$ from the second. Then $g$ is the restriction of $\bar{g}$ to $S^{2 \ell-1}=\partial B^{2 \ell}$.

For the image of $g$ we have, using (2),

$$
g\left(S^{2 \ell-1}\right)=g\left(B^{\ell} \times S^{\ell-1}\right) \cup g\left(S^{\ell-1} \times B^{\ell}\right)=\left(S^{\ell} \times\left\{s_{0}\right\}\right) \cup\left(\left\{s_{0}\right\} \times S^{\ell}\right)=S^{\ell} \vee S^{\ell} .
$$

It remains to observe that $\bar{g}$ restricted to int $B^{2 \ell}$ is a homeomorphism onto $\left(S^{\ell} \times S^{\ell}\right) \backslash$ $\left(S^{\ell} \vee S^{\ell}\right)$. Hence, the result of attaching the boundary of $B^{2 \ell}$ to $S^{\ell} \vee S^{\ell}$ via $g$ is indeed homeomorphic to $S^{\ell} \times S^{\ell}$ as claimed.

Squeezing. Now we define a "squeezing map" from $S^{\ell} \vee S^{\ell}$ to $E$. We let the $\ell$-lollipop $L^{\ell}$ be an $\ell$-dimensional sphere of radius $1 / 2$ with attached segment ("stick") of length 1 ; 


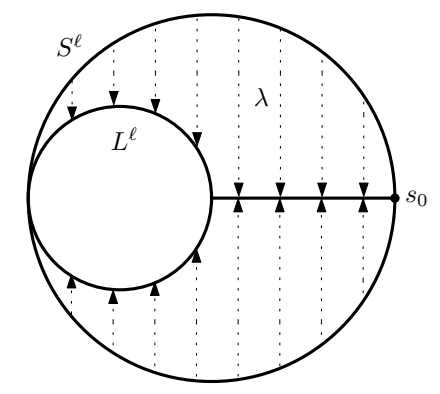

Fig. 10. The map $\lambda$ squeezing $S^{\ell}$ to the lollipop $L^{\ell}$.

see Fig. 10. Formally,

$$
L^{\ell}:=\partial B\left(-s_{0} / 2,1 / 2\right) \cup\left[0, s_{0}\right],
$$

where $B(x, r)$ stands for the ball of radius $r$ centered at $x$. We let $\lambda: S^{\ell} \rightarrow L^{\ell}$ be the projection that moves each point of $S^{\ell}$ in the direction perpendicular to the axis $\left[-s_{0}, s_{0}\right]$.

Now, with $L^{\ell} \vee L^{\ell}:=\left(L^{\ell} \times\left\{s_{0}\right\}\right) \cup\left(\left\{s_{0}\right\} \times L^{\ell}\right)$, we have the map $\lambda \vee \lambda: S^{\ell} \vee S^{\ell} \rightarrow$ $L^{\ell} \vee L^{\ell}$ (given by $\left.(x, y) \mapsto(\lambda(x), \lambda(y))\right)$. Finally, $L^{\ell} \vee L^{\ell}$ can be identified with the complex $E$ as above by a suitable homeomorphism, and we arrive at the map

$$
r=(\lambda \vee \lambda) \circ g: S^{2 \ell-1} \rightarrow E
$$

(where the homeomorphism of $L^{\ell} \vee L^{\ell}$ with $E$ is not explicitly shown).

The clause gadget $X$ is obtained by attaching the boundary of $B^{2 \ell}$ to $E$ via the map $r$. Of course, we want $X$ to be a simplicial complex, and so in reality we use a suitable PL version of the attaching map $r$ (we have not presented it this way since the description above seems more accessible).

For the forthcoming proof of an analogue of Lemma 4.2 , we need the following observation.

Observation 5.2. Let $\kappa: L^{\ell} \vee L^{\ell} \rightarrow S^{\ell} \vee S^{\ell}$ be the quotient map corresponding to contracting the "stick" $c$ of the double lollipop to a single point. Then the composition $\kappa \circ(\lambda \vee \lambda): S^{\ell} \vee S^{\ell} \rightarrow S^{\ell} \vee S^{\ell}$ is homotopic to the identity on $S^{\ell} \vee S^{\ell}$.

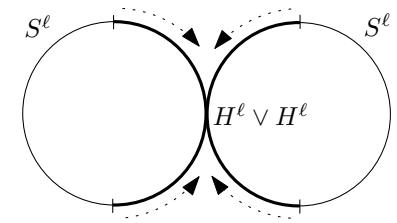

Fig. 11. Contracting the wedge of two hemispheres.

Proof. Let $H^{\ell}:=\left\{x \in S^{\ell}:\left\langle s_{0}, x\right\rangle \geq 0\right\}$ be the closed hemisphere centered at $s_{0}$. The assertion follows by observing that $\kappa \circ(\lambda \vee \lambda)$ is the quotient map corresponding to contracting the subset $H^{\ell} \vee H^{\ell}$ of $S^{\ell} \vee S^{\ell}$ to a single point; see Fig. 11 
Lemma 5.3 (Higher dimensional version of Lemma 4.2).

(i) (Based on [SSS98, Lemma 2.2]) Let $\Sigma_{a}^{\ell}$ and $\Sigma_{b}^{\ell}$ denote the two $\ell$-spheres (boundaries of $(\ell+1)$-simplices) contained in $E \subset X$. Let $S_{a}^{k}$ and $S_{b}^{k}$ be PL k-spheres. Then there is no PL embedding $f$ of the disjoint union $S_{a}^{k} \sqcup S_{b}^{k} \sqcup X$ into $S^{d}$ such that

- the $\ell$-sphere $f\left(\Sigma_{a}^{\ell}\right)$ and the $k$-sphere $f\left(S_{a}^{k}\right)$ have odd linking number, and so do $f\left(\Sigma_{b}^{\ell}\right)$ and $f\left(S_{b}^{k}\right)$;

- $f\left(\Sigma_{a}^{\ell}\right)$ and $f\left(S_{b}^{k}\right)$ are unlinked, and so are $f\left(\Sigma_{b}^{\ell}\right)$ and $f\left(S_{a}^{k}\right)$.

(ii) Let $f$ be a generic linear embedding of $E$ in $\eta^{7} \mathbb{R}^{2 \ell+2}$, and let $\delta>0$. Then there is a PL embedding $\bar{f}$ of $X$ in $\mathbb{R}^{2 \ell+2}$ extending $f$ whose image is contained in the neighborhood $N=N(f, \delta):=N\left(T_{a}, \delta\right) \cup N\left(f\left(\Sigma_{b}^{\ell}\right), \delta\right) \cup N(f(c), \delta)$, where $T_{a}$ is the $(\ell+1)$-dimensional simplex bounded by $f\left(\Sigma_{a}^{\ell}\right)$.

Proof. Part (i) follows from the proof of [SSS98, proof of Lemma 2.2] with only minor modifications. First, before giving a formal proof, we describe the basic approach of [SSS98], which also applies to the proof of Lemma 4.2 (i).

Suppose that a PL embedding $f$ as in (i) above exists. Let $C$ denote the complement $\mathbb{R}^{d} \backslash f\left(S_{a}^{k} \sqcup S_{b}^{k}\right)$, let $r: S^{2 \ell-1} \rightarrow E$ be the attaching map used in the construction of $X$, and let $\bar{r}: B^{2 \ell} \rightarrow X$ be the extension of $r$ to $B^{2 \ell}$ (formally, $\bar{r}$ is the quotient map).

The basic strategy is as follows: On the one hand, using the assumptions about linking numbers, one shows that $f \circ r$ defines a nontrivial element of the homotopy group $\pi_{2 \ell-1}(C)$. On the other hand, $f \circ \bar{r}$ witnesses that $f \circ r$ is homotopically trivial-a contradiction.

As in [SSS98], one distinguishes two cases: $\ell=1$ and $\ell>1$. In the case $\ell=1$, we are dealing with the fundamental group $\pi_{1}(C)$, and the proof is essentially identical to that of [FKT94, Lemma 7], i.e., our Lemma 4.2, which we briefly summarize for the reader's convenience.

To show that $f \circ r: S^{1} \rightarrow C$ is homotopically nontrivial, one first observes that $\pi_{1}(E)$ is the free group on two generators $a$ and $b$, and the attaching map $r: S^{1} \rightarrow E$ corresponds to the commutator $a b a^{-1} b^{-1}$, which is a nontrivial element of $\pi_{1}(E)$. So it suffices to show that the map $f_{\star}: \pi_{1}(E) \rightarrow \pi_{1}(C)$ induced by the restriction $\left.f\right|_{E}$ is injective. To this end, one first considers the homomorphisms $f_{* 1}$ and $f_{* 2}$ induced by $\left.f\right|_{E}$ in the first and second homology.

By Alexander duality, the complement $C$ has the same homology (with $\mathbb{Z}_{2}$ coefficients, say) as $S^{1} \vee S^{1}$, and thus $H_{1}\left(C ; \mathbb{Z}_{2}\right)=\mathbb{Z}_{2} \oplus \mathbb{Z}_{2}$ and $H_{2}\left(C ; \mathbb{Z}_{2}\right)=0$. For $E$ we have $H_{1}\left(E ; \mathbb{Z}_{2}\right) \cong \mathbb{Z}_{2} \oplus \mathbb{Z}_{2}$ with a basis represented by the two circles $\Sigma_{a}^{1}$ and $\Sigma_{b}^{1}$. The assumption on the linking numbers implies that $f_{* 1}$ is an isomorphism, and $f_{* 2}$ is trivially surjective. Then the injectivity of the homomorphism $f_{\star}$ of the fundamental groups follows from a theorem of Stallings [Sta65], which finishes the case $\ell=1$.

\footnotetext{
7 It follows from our assumptions on $d$ and $k$ that $d \geq 2 \ell+3$. Therefore, when, in the course of the reduction, we construct an embedding of a complex associated with a satisfiable formula, we can afford to embed each conflict gadget in its own "private" $(2 \ell+2)$-dimensional set. Since two $\ell$-spheres in dimension $2 \ell+2$ are never linked, we do not need to make an explicit unlinking assumption as in Lemma 4.2
} 
In the case $\ell>1$, the proof that $f \circ r$ defines a nontrivial element of $\pi_{2 \ell-1}(C)$ requires somewhat more advanced machinery. Segal et al. [SSS98] prove essentially the same assertion as in part (i) of the lemma, with the following differences:

1. $X$ is replaced by $X^{\prime}$, which is obtained by attaching $B^{2 \ell}$ to $\Sigma_{a}^{\ell} \vee \Sigma_{b}^{\ell}$ via the map $g$ (as described above) and hence homeomorphic to $S^{\ell} \times S^{\ell}$.

2. The disjoint union $S_{a}^{k} \sqcup S_{b}^{k}$ is replaced by the wedge $8 S_{a}^{k} \vee S_{b}^{k}$.

They show that if there were an embedding $f$ of $\left(\Sigma_{a}^{\ell} \vee \Sigma_{b}^{\ell}\right) \sqcup\left(S_{a}^{k} \vee S_{b}^{k}\right)$ with the linking properties as in part (i) of the lemma, $f \circ g$ would be a nontrivial element of $\pi_{2 \ell-1}(C)$.

Now we begin with a formal proof of Lemma 5.3. Instead of modifying the proof of [SSS98], we show how to reduce our assertion to theirs. Suppose there were a bad embedding $f$ of $S_{a}^{k} \sqcup S_{b}^{k} \sqcup X$ as in the lemma. Since the codimension of the image $f\left(S_{a}^{k} \sqcup S_{b}^{k} \sqcup X\right)$ is at least 2, we can grow a $k$-dimensional finger from $f\left(S_{a}^{k}\right)$ towards $f\left(S_{b}^{k}\right)$ avoiding $f(X)$ until the finger touches $f\left(S_{b}^{k}\right)$ in a single point. This results in an embedding of $\left(S_{a}^{k} \vee S_{b}^{k}\right) \sqcup X$. For simplicity, we denote this modified embedding by $f$ as well.

We observe that when pulling the finger, we can pull along a $(k+1)$-dimensional image of $B^{k+1}$ filling $f\left(S_{a}^{k}\right)$, and so the images are still linked or unlinked as in the assumption of the lemma.

Next, consider the image $f(E)$ of the double lollipop in $C$. We modify $f$ as follows. We deformation-retract the arc $f(c)$ to its midpoint $m$, pulling along $\ell$-dimensional fingers from the two $\ell$-spheres $f\left(\Sigma_{a}^{\ell}\right)$ and $f\left(\Sigma_{b}^{\ell}\right)$, so that at the end of the deformation, the fingers touch in the single point $m$. This describes a continuous deformation of $\left.f\right|_{E}$ that only changes $\left.f\right|_{E}$ on the segment $c$ and in two small neigborhoods $U_{a}$ and $U_{b}$ of the endpoints of $c$ in the $\ell$-spheres (these neighborhoods provide the "material" for the fingers). We have to take care to pull along the parts of $B^{2 \ell}$ attached to $U_{a}$ and $U_{b}$, respectively, i.e., we extend the deformation to a continuous deformation of $f$ on all of $X$ that changes $f$ only on a small neighborhood $V$ in $X$ of $c \cup U_{a} \cup U_{b}$. The whole deformation can be carried out so that the image of $V$ remains in a small $\varepsilon$-neighborhood of the original image $f(E)$ throughout the deformation. Let $f^{\prime}$ be the final modified map from $S_{a}^{k} \sqcup S_{b}^{k} \sqcup X$ into $S^{d}$ (note that we made no changes on the two $k$-spheres). The map $f^{\prime}$ maps the "bent stick" $c$ of the double lollipop constantly to $m$ (in particular, it is not an embedding), and it induces a unique embedding $f^{\prime \prime}: X^{\prime} \rightarrow C$ such that $f^{\prime \prime}$ agrees with $f^{\prime}$ on the interior of $B^{2 \ell}$ and $f^{\prime \prime} \circ \kappa=f^{\prime}$ on $E$, where $\kappa$ is the map from Observation 5.2 Moreover, the map $f \circ r=f \circ(\lambda \vee \lambda) \circ g: S^{2 \ell-1} \rightarrow C$ is deformed into the map $f^{\prime \prime} \circ \kappa \circ(\lambda \vee \lambda) \circ g: S^{2 \ell-1} \rightarrow C$. Thus, $f \circ r$ and $f^{\prime \prime} \circ \kappa \circ(\lambda \vee \lambda) \circ g$ define the same element of $\pi_{2 \ell-1}(C)$. However, by Observation 5.2, the latter map is homotopic to $f^{\prime \prime} \circ g$. Thus, $f \circ r$ and $f^{\prime \prime} \circ g$ define the same element of $\pi_{2 \ell-1}(C)$. But the former is trivial, as witnessed by $f \circ \bar{r}$, while the latter is not according to [SSS98] - a contradiction. This completes the proof of (i).

We now turn to the proof of (ii). For easier presentation, we describe an embedding $\bar{f}$ that is not a priori PL; it is routine to replace it by a PL embedding.

\footnotetext{
8 Wedges are used for technical reasons: By a theorem of Lickorish [Lic65], any embedding (PL or even topological) of a wedge of spheres of codimension at least 3 is unknotted, i.e., ambient isotopic to a standard embedding.
} 
Applying a suitable homeomorphism $\mathbb{R}^{2 \ell+2} \rightarrow \mathbb{R}^{2 \ell+2}$, we may assume that $f(E)$ is actually $L^{\ell} \vee L^{\ell}$. Let $\bar{L}^{\ell}$ denote the $\ell$-lollipop with its $\ell$-sphere filled (i.e., $\bar{L}^{\ell}:=$ $\left.B\left(-s_{0} / 2,1 / 2\right) \cup\left[0, s_{0}\right]\right)$. It suffices to embed $X$ in the $\delta$-neighborhood of $L^{\ell} \vee \bar{L}^{\ell}$ for $\delta>0$ arbitrarily small; actually, for notational convenience, we will eventually get $4 \delta$ instead of $\delta$.

Instead of specifying the embedding $\bar{f}: X \rightarrow \mathbb{R}^{2 \ell+2}$ directly, we define a mapping $\tilde{f}: S^{\ell} \times S^{\ell} \rightarrow \mathbb{R}^{2 \ell+2}$ that coincides with $\lambda \vee \lambda$ on $S^{\ell} \vee S^{\ell}$ and maps the rest of $S^{\ell} \times S^{\ell}$ homeomorphically. Then $\bar{f}$ can be given as (considering $E$ identified with $L^{\ell} \vee L^{\ell}$ )

$$
\bar{f}(z)= \begin{cases}z & \text { for } z \in E, \\ \tilde{f}(z) & \text { for } z \notin E .\end{cases}
$$

Writing a point of $S^{\ell} \times S^{\ell}$ as $(x, y)$, we define $\tilde{f}$ using two auxiliary maps $u, v: S^{\ell} \times$ $[0, \infty) \rightarrow \mathbb{R}^{\ell+1}$ :

$$
\tilde{f}(x, y):=\left(u\left(x, \operatorname{dist}\left(y, s_{0}\right)\right), v\left(y, \operatorname{dist}\left(x, s_{0}\right)\right)\right) .
$$

To define $u(x, t)$, we think of $t$ as time. For $t=0$, the image $u\left(S^{\ell}, t\right)$ is the lollipop $L^{\ell}$, while for all $t>0$ it is topologically a sphere, which looks almost like the lollipop; see Fig. 12. Concretely, we set

$$
u(x, t):= \begin{cases}t x+(1-t) \lambda(x) & \text { for } 0 \leq t \leq \delta \\ \delta x+(1-\delta) \lambda(x) & \text { for } t \geq \delta .\end{cases}
$$
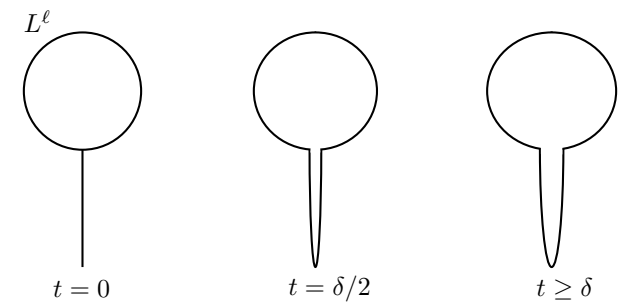

Fig. 12. The (images of the) mappings $u(*, t)$.
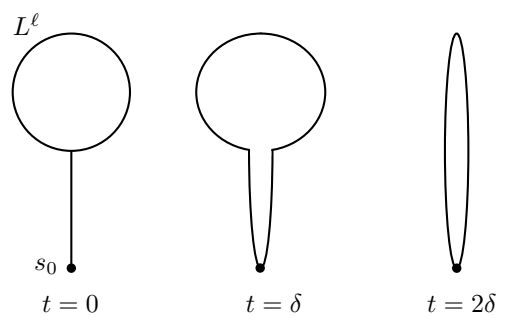

Fig. 13. The (images of the) mappings $v(*, t)$.

As for $v$, we let it coincide with $u$ for $t \leq \delta$ (see Fig. 13). For $t=2 \delta$, we set $v(x, 2 \delta):=$ $\left(x_{1}, \delta x_{2}, \delta x_{3}, \ldots, \delta x_{\ell+1}\right)$, and for all $t \geq 3 \delta$ we set $v(x, t):=\delta\left(x-s_{0}\right)+s_{0}$ (i.e., the 
sphere is shrunk by a factor of $\delta$ so that it still touches $s_{0}$ ). On the intervals $[\delta, 2 \delta]$ and $[2 \delta, 3 \delta]$ we interpolate $v(x, t)$ linearly in $t$.

The $\bar{f}$ defined in this way is clearly continuous and coincides with $\lambda \vee \lambda$ on $S^{\ell} \vee S^{\ell}$. Next, we want to show $\bar{f}(x, y) \neq \bar{f}\left(x^{\prime}, y^{\prime}\right)$ whenever $(x, y) \neq\left(x^{\prime}, y^{\prime}\right)$ and none of $x, x^{\prime}, y, y^{\prime}$ equals $s_{0}$. First we note that $u(x, t) \neq u\left(x^{\prime}, t^{\prime}\right)$ whenever $x \neq x^{\prime}$ and $t, t^{\prime}>0$, and thus we may assume $x=x^{\prime}, y \neq y^{\prime}$. Then we just use the injectivity of $v(*, t)$ for every $t>0$.

It remains to check that the image of $\bar{f}$ lies close to $\bar{L}^{\ell} \vee L^{\ell}$. The image $u\left(S^{\ell}, t\right)$ is $\delta$-close to $L^{\ell}$ for all $t$, and the image $v\left(S^{\ell}, t\right)$ is $2 \delta$-close to $s_{0}$ whenever $t \geq 3 \delta$. Thus, whenever $\operatorname{dist}\left(x, s_{0}\right) \geq 3 \delta$, we have $\bar{f}(x, y)$ lying $3 \delta$-close to $L^{\ell} \times\left\{s_{0}\right\}$.

Next, let us assume dist $\left(x, s_{0}\right) \leq 3 \delta$. Then $u(x, t)$ is $3 \delta$-close to $s_{0}$ for all $t$, and observing that $v(y, t)$ always lies $\delta$-close to the filled lollipop $\bar{L}^{\ell}$, we conclude that $\bar{f}(x, y)$ is $4 \delta$-close to $\left\{s_{0}\right\} \times \bar{L}^{\ell}$.

\subsection{The reduction}

Having introduced the clause gadget and the conflict gadget, the rest of the reduction is almost the same as in Section 4.3. and so we mainly point out the (minor) differences.

Given a 3-CNF formula $\varphi$, the simplicial complex is pasted together from the gadgets exactly as in Section 4.3, we have $\operatorname{dim} K=\max (k, 2 \ell)=k$. For $\varphi$ unsatisfiable, the nonembeddability of $K$ is shown using Lemmas 5.1 (i) and 5.3 (i) instead of Lemmas 4.1 (i) and 4.2 (i), but otherwise in the same way as in Section 4.3 .

Given a satisfiable formula $\varphi$, we again begin by embedding the clause gadgets, this time using Lemma 5.1(ii). For an opening $\omega$ of a clause gadget $G_{i}$, we can again obtain a set $Q_{\omega} \subset \mathbb{R}^{d}$ with $Q_{\omega} \cap G_{i}=\Sigma$, where $\Sigma=f(\partial \omega)$, this time homeomorphic to $T^{\varepsilon} \times B^{k}$ (where $T$ is the $(\ell+1)$-dimensional simplex bounded by $\Sigma$ and $T^{\varepsilon}$ is the part of it $\varepsilon$-close to $\Sigma$ ). Similarly we can build, for a non-witness opening $\omega$, the set $Q_{\omega}^{+}$homeomorphic to $T \times B^{k}$.

Now we need to define the "private pieces" $Q_{\omega j}$ and $Q_{\omega j}^{+}, j=1, \ldots, t$, within each $Q_{\omega}$ and $Q_{\omega}^{+}$, respectively. This time we first choose pairwise disjoint sets $B_{1}, \ldots, B_{t} \subset$ $\partial B^{k}$, each homeomorphic to $B^{\ell+1}$ (for this we need $k \geq \ell+2$, which holds in our setting), we let $W_{j}$ be the cone with base $B_{j}$ and apex at the center of $B^{k}$, and we let $w_{j}$ be the boundary of $W_{j}$ (not including the interior of the base $B_{j}$ ). We have $W_{j}$ homeomorphic to $B^{\ell+2}$ and $w_{j}$ to $B^{\ell+1}$, and this allows us to construct $Q_{\omega j}$ homeomorphic to a $(2 \ell+$ 2)-dimensional neighborhood of $\Sigma$, and $Q_{\omega j}^{+}$homeomorphic to a $(2 \ell+2)$-dimensional neighborhood of $T$.

The rest of the embedding construction can be copied from Section 4.3 almost verbatim. This concludes the proof of Theorem 1.3 .

\section{Linkless embeddings}

A PL embedding $f$ of a graph $G$ into $\mathbb{R}^{3}$ is called linkless if the images of any two vertexdisjoint cycles in $G$ are unlinked, i.e., each of them bounds a PL disk that is disjoint from the other. 
Robertson, Seymour, and Thomas [RST93, RST95] showed, establishing a conjecture of Sachs, that a finite graph $G$ is linklessly embeddable in $\mathbb{R}^{3}$ if and only if $G$ contains none of the seven graphs in the so-called Petersen family as a minor. Moreover, they show (confirming a conjecture by Böhme [Böh90]) that every linklessly embeddable graph $G$ has even a panelled embedding (also called a flat embedding in some sources) into $\mathbb{R}^{3}$, i.e., a PL embedding such that for every cycle $C$ in $G$ there exists a PL disk $D$ in $\mathbb{R}^{3}$ whose boundary equals $f(C)$ and that is otherwise disjoint from $f(G)$. It follows from the forbidden minor criterion that linkless embeddability, as well as panelled embeddability, can be tested in polynomial time (although the algorithm does not find an embedding); see [RST93, RST95].

The following lemma can be used to relate panelled embeddability to embeddability of 2-dimensional complexes into $\mathbb{R}^{3}$.

Lemma 6.1 (Böhme [B̈̈h90]). Let $f$ be a panelled embedding of $G$ into $\mathbb{R}^{3}$, and let $C_{1}, \ldots, C_{m}$ be a family of cycles in $G$ any two of which are either disjoint or intersect in a path. Then there exist PL disks $D_{1}, \ldots, D_{m}$ in $\mathbb{R}^{3}$ such that $\partial D_{i}=f\left(C_{i}\right)$ and the interiors of the $D_{i}$ are pairwise disjoint and disjoint from $f(G)$.

Corollary 6.2. Let $K$ be a 2-dimensional simplicial complex whose 1-skeleton does not have a minor from the Petersen family (and thus is linklessly embeddable). Then $\mathrm{K}$ embeds in $\mathbb{R}^{3}$.

Proof. If $G$ is the 1-skeleton of $K$, then the boundaries of the triangles in $K$ form a family of cycles as in Lemma 6.1. Hence a panelled embedding of $G$ can be extended to an embedding of $K$.

We note that the general problem $\mathrm{EMBED}_{2 \rightarrow 3}$ can be rephrased as a partially panelled embedding problem for graphs, whose input is a graph $G$ and a family of triangles $C_{1}, \ldots, C_{m}$ in $G$, and the question is whether $G$ admits a PL embedding in which each $C_{i}$ can be panelled. This in itself does not tell us anything new about the computational complexity of the problem, of course.

\section{Appendix A. A decision algorithm for $\mathrm{EMBED}_{2 \rightarrow 2}$ (sketch)}

Given a 2-dimensional simplicial complex $K$, we want to test whether it is embeddable in $\mathbb{R}^{2}$. To this end, we can use a characterization of 2-dimensional simplicial complexes embeddable in $\mathbb{R}^{2}$ due to Halin and Jung [HJ64]. They give a list of seven small simplicial complexes, denoted by $K_{\mathrm{I}}$ through $K_{\mathrm{VII}}$ and shown in Fig. 14, such that a 2-dimensional simplicial complex $K$ is embeddable in $\mathbb{R}^{2}$ iff it does not contain a subdivision of some of $K_{\mathrm{I}}-K_{\mathrm{VIII}}$ as a subcomplex.

An inspection of $K_{\mathrm{I}}-K_{\mathrm{VIII}}$ reveals that they are of three basic types:

(a) $K_{\text {III }}$ is homeomorphic to $S^{2}$.

(b) $K_{\mathrm{VI}}$ is a "disk with a stick", i.e. a subdivision of a triangle with an edge attached to a vertex in the middle. 


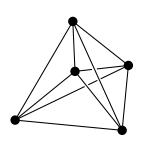

$K_{\mathrm{I}} \cong K_{5}$

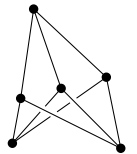

$K_{\mathrm{II}} \cong K_{3,3}$

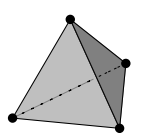

$K_{\text {III }} \cong S^{2}$
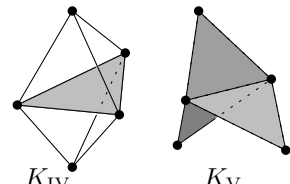

$K_{\mathrm{V}}$

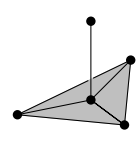

$K_{\mathrm{VI}}$

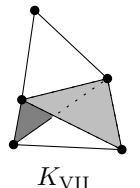

$K_{\mathrm{VII}}$

Fig. 14. The forbidden subcomplexes $K_{\mathrm{I}}-K_{\mathrm{VII}}$.

(c) Each of the remaining five types contains a subgraph isomorphic to $K_{3,3}$ or $K_{5}$ in the 1-skeleton or in the 1-skeleton of the first barycentric subdivision (see Fig. 15 for the latter cases).

(A slightly different characterization of 2-dimensional complexes embeddable in $S^{2}$ can be found in Mardešić and Segal [MS66]; it is somewhat less convenient for our purposes.)

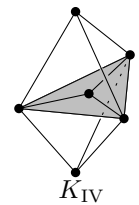

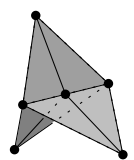

$K_{\mathrm{V}}$

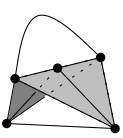

$K_{\mathrm{VII}}$

Fig. 15. $K_{\mathrm{IV}}$ and $K_{\mathrm{V}}$ contain $K_{3,3}$, and $K_{\mathrm{VII}}$ contains $K_{5}$.

Thus, the following algorithm decides the embeddability of a given 2-dimensional complex $K$ into $\mathbb{R}^{2}$ :

First, test if the 1-skeleton of the first barycentric subdivision of $K$ is a planar graph (this takes care of (c)), and test if the link of each vertex of $K$ is either acyclic or consists of a single cycle (this deals with (b)).

If both tests yield a positive answer, we know that either $K$ embeds into the plane, or it contains a subdivision of $K_{\text {III }}$, i.e., a 2-dimensional sphere. Moreover, since $K_{\mathrm{V}}$ is already excluded as a subcomplex, every edge of $K$ is incident to at most two triangles. This allows us to test in linear time if $K$ contains a homological cycle with $\mathbb{Z}_{2}$ coefficients: Consider the dual graph of $K$, whose vertices are the triangles of $K$, and two triangles are adjacent if they share an edge. Since every edge of $K$ is incident to at most two triangles, every inclusion-minimal homological cycle is an entire connected component of triangles in the dual graph.

Starting from an arbitrary triangle, we can find its component in linear time (using depth-first-search, say). If some triangle in the component has a free edge with no other triangle incident to it, we can discard the entire component and start again. If we can discard all components of triangles in this way, we have also excluded (a), and consequently $K$ is planar. Otherwise, we have found a collection $C$ of triangles in $K$ such that every edge is incident to either 0 or 2 triangles of $C$, i.e., a homological cycle, which witnesses that $K$ does not embed in $\mathbb{R}^{2}$ (in fact, by the characterization of Halin and Jung, and since (b) and (c) are already excluded, we know that any minimial homological cycle we find must actually be a 2 -dimensional sphere). 
These tests can be performed in linear time. We remark that this only gives a decision algorithm and does not actually construct an embedding. Daniel Král' (personal communication) has independently devised a linear-time algorithm, which moreover produces an embedding.

\section{Appendix B. The deleted product obstruction}

Here we recall the Haefliger-Weber theorem and some related material.

Let $X$ be a topological space. The deleted product of $X$, which we denote by $X_{\Delta}^{2}$, is the following subspace of the Cartesian product $X \times X$ :

$$
X_{\Delta}^{2}:=X \times X \backslash\{(x, x): x \in X\} .
$$

An embedding $f: X \rightarrow \mathbb{R}^{d}$ induces a continuous map $\tilde{f}: X_{\Delta}^{2} \rightarrow S^{d-1}$ by

$$
\tilde{f}(x, y):=\frac{f(x)-f(y)}{\|f(x)-f(y)\|} .
$$

Moreover, this map is equivariant, which in this particular case means that $\tilde{f}(y, x)=$ $-\tilde{f}(x, y)$ for all $(x, y) \in X_{\Delta}^{2}$. Thus, the existence of an equivariant map of $X_{\Delta}^{2}$ in $S^{d-1}$ is necessary for embeddability of $X$ into $\mathbb{R}^{d}$.

The Haefliger-Weber theorem shows that, surprisingly, this necessary condition is also sufficient if $X$ is (the polyhedron of) a $k$-dimensional simplicial complex and $k$ lies in the metastable range (mentioned after Theorem 1.3$)$, namely, for $k \leq(2 d-3) / 3$. See Haefliger [Hae64] and Weber [Web67] for original sources and, e.g., Skopenkov [Sko06] for a modern overview, proof sketch, and extensions. Thus, for example, for $(k, d)=$ $(3,6),(4,8),(5,9)$, etc., the embeddability of a given $k$-dimensional simplicial complex into $\mathbb{R}^{d}$ is equivalent to the existence of an equivariant map of the space $|K|_{\Delta}^{2}$ into $S^{d-1}$.

For the case $d=2 k$, the existence of such an equivariant map can be decided using the first (equivariant) cohomological obstruction, which in this particular case is equivalent to the Van Kampen obstruction mentioned in Appendix D

As was suggested by one of the referees, for the case $d=2 k-1$, the existence of an equivariant map is characterized by (an equivariant version of) a theorem of Steenrod [Ste47], which involves the first cohomological obstruction plus another apparently computable invariant (a Steenrod square operation in cohomology). This might also yield even a polynomial-time decision algorithm, but some computational issues still need to be checked.

For general $k$ and $d$, the existence of an equivariant map $|K|_{\Delta}^{2} \rightarrow S^{d-1}$ can be detected, e.g., via the extraordinary Van Kampen obstruction defined in Melikhov [Mel06]. However, the computability of this obstruction remains to be clarified. More generally, the computation of the cohomotopy set $\left[K, S^{m}\right]$ for a simplicial complex $K$, or its equivariant version (which is what we could use for the embeddability question), might be possible by "dualizing" the methods for computing higher homotopy groups mentioned in the introduction.

Examples of incompleteness of the deleted product condition. It is known that the condition $k \leq(2 d-3) / 3$ defining the metastable range in the Haefliger-Weber theorem 
is sharp, in the following sense: For each pair $(k, d)$ with $d \geq 3$ and $d \geq k>(2 d-3) / 3$, there exists a finite simplicial complex $K$ of dimension (at most) $k$ that is not PL embeddable in $\mathbb{R}^{d}$, yet such that an equivariant map $|K|_{\Delta}^{2} \rightarrow S^{d-1}$ exists. This was proved in several papers, dealing with various values of $(k, d)$ : Mardešić and Segal [MS67] $((d, d)$ for every $d \geq 4$ ), Segal and Spież [SS92] (all values outside the metastable range with finitely many exceptions), Freedman, Krushkal, Teichner [FKT94] $(k=2, d=4)$, Segal, Skopenkov, and Spież [SSS98] (all remaining cases except for $(k, d)=(2,3),(3,3))$, and Gonçalves and Skopenkov [GS06] $((k, d)=(2,3),(3,3))$.

\section{Appendix C. PL embeddings versus topological embeddings}

Let us say that TOP and PL embeddability coincide for $(k, d)$ if every finite simplicial complex of dimension at most $k$ that can be topologically embedded in $\mathbb{R}^{d}$ can also be PL embedded in $\mathbb{R}^{d}$.

The Alexander horned sphere and the other examples mentioned in Section 2 show that topological embeddings may exhibit behavior that is impossible for PL embeddings. However, they do not clarify the relation of the class of $k$-dimensional complexes PL embeddable in $\mathbb{R}^{d}$, i.e., the YES instances of $\mathrm{EMBED}_{k \rightarrow d}$, to the class of $k$-dimensional complexes topologically embeddable in $\mathbb{R}^{d}$. If these two classes coincide, we will say that TOP and PL embeddability coincide for $(k, d)$.

On the positive side, it is known that TOP and PL embeddability coincide for $(k, d)$ whenever $d-k \geq 3$ [Bry72], and also for $(k, d)=(2,3)$. The latter follows from Theorem 5 of Bing [Bin59], which shows that the image of a topological embedding of a 2-dimensional complex in $\mathbb{R}^{3}$ is homeomorphic to a polyhedron PL embedded in $\mathbb{R}^{3}$, and from a result of Papakyriakopoulos [Pap43] ("Hauptvermutung" for 2-dimensional polyhedra) that any two 2-dimensional polyhedra that are homeomorphic are also PL homeomorphic.

However, TOP and PL embeddability do not always coincide: there is an example of a 4-dimensional complex (namely, the suspension of the Poincaré homology 3-sphere) that embeds topologically, but not PL, into $\mathbb{R}^{5}$. For this example we are indebted to Colin Rourke (private communication); unfortunately, his proof, although short, uses too advanced concepts to be reproduced here. It would be interesting to clarify in general for what $(k, d)$ TOP and PL embeddability coincide.

Let us also mention that simplicial complexes do not capture all "finitary" topological spaces whose embeddability one might want to investigate. An interesting example is Freedman's $E_{8}$ manifold [Fre82], which is a 4-dimensional compact topological manifold that is not homeomorphic to the polyhedron of any simplicial complex.

\section{Appendix D. The Van Kampen obstruction: An algorithmic perspective}

The algorithm for deciding $\mathrm{EMBED}_{k \rightarrow 2 k}, k \neq 2$. Let $K$ be a $k$-dimensional finite simplicial complex. We are going to define an object associated with $K$, the Van Kampen obstruction $\mathfrak{o}_{K}$. 
Let

$$
P:=\{(\sigma, \tau): \sigma, \tau \in K, \sigma \cap \tau=\emptyset, \operatorname{dim} \sigma=\operatorname{dim} \tau=k\}
$$

be the set of all ordered pairs of disjoint $k$-dimensional simplices of $K$. From a rather pedestrian point of view, which we mostly adopt here, $\mathfrak{o}_{K}$ is a subset of $\mathbb{Z}^{P}$, i.e., a set of integer vectors with components indexed by $P$ (in the language of algebraic topology, $\mathfrak{o}_{K}$ is an element of a certain cohomology group). First we give a simple combinatorial description, and later on we will offer a geometric interpretation, which will also explain some of the terminology.

To define $\mathfrak{o}_{K}$ we need to fix some (arbitrary) linear ordering $\leq$ of the vertices of $K$ (although the final result does not depend on it). We will write a $k$-dimensional simplex $\sigma \in K$ as $\sigma=\left[v_{0}, v_{1}, \ldots, v_{k}\right]$, meaning that $v_{0}$ through $v_{k}$ are the vertices of $\sigma$ in increasing order under $\leq$.

First we define one particular vector $o_{\gamma}$ of $\mathfrak{o}_{K}$. The component corresponding to a pair $(\sigma, \tau) \in P$, where $\sigma=\left[v_{0}, v_{1}, \ldots, v_{k}\right]$ and $\tau=\left[w_{0}, w_{1}, \ldots, w_{k}\right]$, is

$$
\left(o_{\gamma}\right)_{\sigma, \tau}:= \begin{cases}+1 & \text { if } v_{0}<w_{0}<v_{1}<w_{1}<\cdots<v_{k}<w_{k}, \\ (-1)^{k} & \text { if } w_{0}<v_{0}<w_{1}<v_{1}<\cdots<w_{k}<v_{k}, \\ 0 & \text { otherwise. }\end{cases}
$$

Next, we define a set $\Phi \subseteq \mathbb{Z}^{P}$, whose elements we will call the finger move vectors. The vectors in $\Phi$ correspond to pairs $(\omega, v)$ of simplices in $K$ such that one of $\omega, v$ has dimension $k$ and the other one has dimension $k-1$. Formally, we set

$$
\begin{aligned}
& Q:=\{(\omega, v): \omega, v \in K, \omega \cap v=\emptyset, \operatorname{dim} \omega+\operatorname{dim} v=2 k-1\}, \\
& \Phi:=\left\{\varphi^{\omega, v}:(\omega, v) \in Q\right\}
\end{aligned}
$$

To define the component $\varphi_{\sigma, \tau}^{\omega, v}$, we again write $\sigma=\left[v_{0}, v_{1}, \ldots, v_{k}\right]$ and $\tau=$ $\left[w_{0}, w_{1}, \ldots, w_{k}\right]$. We set

$$
\varphi_{\sigma, \tau}^{\omega, v}:= \begin{cases}(-1)^{i} & \text { if } v=\tau, \omega=\left[v_{0}, v_{1}, \ldots, v_{i-1}, v_{i+1}, \ldots, v_{k}\right] \\ (-1)^{i+k} & \text { if } \omega=\sigma, v=\left[w_{0}, w_{1}, \ldots, w_{i-1}, w_{i+1}, \ldots, w_{k}\right] \\ (-1)^{i+k} & \text { if } \omega=\tau, v=\left[v_{0}, v_{1}, \ldots, v_{i-1}, v_{i+1}, \ldots, v_{k}\right] \\ (-1)^{i} & \text { if } v=\sigma, \omega=\left[w_{0}, w_{1}, \ldots, w_{i-1}, w_{i+1}, \ldots, w_{k}\right]\end{cases}
$$

Thus, $\varphi_{\sigma, \tau}^{\omega, v}$ is nonzero only if the $(k-1)$-dimensional simplex among $\omega, v$ is a facet of one of $\sigma, \tau$ and the $k$-dimensional simplex among $\omega, v$ equals the other.

Now, finally, we can define $\mathfrak{o}_{K}$ as the set of all vectors that can be obtained from $o_{\gamma}$ by adding integer linear combinations of vectors in $\Phi$; formally, $\mathfrak{o}_{K}:=o_{\gamma}+\operatorname{span}_{\mathbb{Z}}(\Phi)$. We say that $\mathfrak{o}_{K}$ vanishes if it contains the zero vector.

We have the following result, based on ideas of [vK32] and proved independently by Shapiro [Sha57] and Wu [Wu65], although their description of the Van Kampen obstruction is different from ours:

Theorem D.1. Let $K$ be a finite $k$-dimensional simplicial complex, $k \neq 2$. Then $\mathfrak{o}_{K}$ vanishes if and only if $K$ can be PL embedded in $\mathbb{R}^{2 k}$. 
This theorem provides a decision algorithm for $\mathrm{EMBED}_{k \rightarrow 2 k}, k \neq 2$. Indeed, it is clear from the above description that, given $K$, we can set up $o_{\gamma}$ and $\Phi$ in polynomial time. The question of whether $\mathfrak{o}_{K}$ vanishes amounts to testing whether $o_{\gamma}$ is an integer linear combination of vectors in $\Phi$. This can be done by bringing the matrix with the vectors of $\Phi$ as columns to the Smith normal form, for which several polynomial-time algorithms are available in the literature. The asymptotically fastest deterministic algorithm to date seems to be the one given in [Sto96]; fast randomized algorithms were given, for instance, in [Gie01, DSV01]. The latter seem to be particularly efficient in the case of sparse matrices.

\section{Remarks.}

1. If $\mathfrak{o}_{K}$ does not vanish, then $K$ does not embed into $\mathbb{R}^{2 k}$ even topologically [Sha57], [Wu65].

2. If $\mathfrak{o}_{K}$ vanishes, the Van Kampen approach also constructs a PL embedding. However, nothing seems to be known about the complexity of the resulting embedding (the number of simplices in a subdivision of $K$ on which the embedding is linear), and it appears that a straightforward implementation of the construction may lead to an at least exponential complexity in the worst case.

3. The case $k=2$ is indeed an exception; the point of [FKT94] is to provide a 2dimensional simplicial complex $K$ that is not embeddable in $\mathbb{R}^{4}$, yet such that $\mathfrak{o}_{K}$ vanishes.

4. The various sign rules in the definition of the Van Kampen obstruction are rather unpleasant; indeed, as pointed out by Melikhov [Mel06], a number of papers on the Van Kampen obstruction (including [FKT94]) contain a sign error. It would be much easier to work over $\mathbb{Z}_{2}$ instead of over the integers (i.e., to reduce all components of the vectors modulo 2); then instead of using the Smith normal form, we could simply solve a system of linear equations. However, an analog of Theorem D.1 for the Van Kampen obstruction reduced modulo 2 is valid only for $k=1$ (where better planarity algorithms exist anyway), while for all $k \geq 3$ Melikhov [Mel06] provides examples of $k$-dimensional complexes that are not embeddable in $\mathbb{R}^{2 k}$ but whose Van Kampen obstruction modulo 2 vanishes.

5. The Van Kampen obstruction can also be defined for embedding of $k$-dimensional complexes into $\mathbb{R}^{d}, k \leq d \leq 2 k$. The definition is similar to the one given above but formally more complicated (especially concerning the various signs). We suspect (although we have no examples or references at present) that the obstruction is incomplete whenever $d<2 k$; that is, that there are nonembeddable complexes for which the obstruction vanishes. However, it is still true that nonvanishing Van Kampen obstruction implies nonembeddability, and so we have a potentially useful tool for excluding many nonembeddable complexes.

6. From the point of view of algebraic topology, $\mathfrak{o}_{K}$ can be regarded as the first obstruction in integral cohomology to the existence of an equivariant map of the deleted product of $K$ into $S^{2 k-1}$ (see Appendix B. As such it is an element of a certain equivariant cohomology group. See, e.g., [Mel06]. 
A geometric view of $\mathfrak{o}_{K}$. Let $f$ be a generic linear mapping of a finite $k$-dimensional simplicial complex into $\mathbb{R}^{2 k}$. Let us consider a pair of disjoint $k$-dimensional simplices $(\sigma, \tau) \in P, \sigma=\left[v_{0}, v_{1}, \ldots, v_{k}\right], \tau=\left[w_{0}, w_{1}, \ldots, w_{k}\right]$. The images $f(\sigma)$ and $f(\tau)$ are $k$-dimensional simplices in $\mathbb{R}^{2 k}$, and because of genericity they either are disjoint or intersect in a single point. Moreover, again by genericity, the union of their vertex sets is in general position and thus

$$
\begin{aligned}
B:=\left(f\left(v_{1}\right)-f\left(v_{0}\right), f\right. & f\left(v_{2}\right)-f\left(v_{0}\right), \ldots, f\left(v_{k}\right)-f\left(v_{0}\right), \\
& \left.f\left(w_{1}\right)-f\left(w_{0}\right), f\left(w_{2}\right)-f\left(w_{0}\right), \ldots, f\left(w_{k}\right)-f\left(w_{0}\right)\right)
\end{aligned}
$$

is an (ordered) basis of $\mathbb{R}^{2 k}$. We define the intersection number of $f(\sigma)$ and $f(\tau)$ as

$$
f(\sigma) \cdot f(\tau):= \begin{cases}+1 & \text { if } f(\sigma) \cap f(\tau) \neq \emptyset \text { and } B \text { is positively oriented, } \\ -1 & \text { if } f(\sigma) \cap f(\tau) \neq \emptyset \text { and } B \text { is negatively oriented, } \\ 0 & \text { otherwise. }\end{cases}
$$

Considering how permuting a basis influences its orientation, one can check the equality $f(\tau) \cdot f(\sigma)=(-1)^{k} f(\sigma) \cdot f(\tau)$. Then we define a vector $o_{f} \in \mathbb{Z}^{P}$ by

$$
\left(o_{f}\right)_{\sigma, \tau}:=(-1)^{k} f(\sigma) \cdot f(\tau) .
$$

For reasons indicated later on, for every $f$ we have $o_{f} \in \mathfrak{o}_{K}$. The $o_{\gamma}$ above corresponds to a particular choice of $f$. Namely, for $t \in \mathbb{R}$, let

$$
\gamma(t):=\left(t, t^{2}, \ldots, t^{2 k}\right) \in \mathbb{R}^{2 k}
$$

the set $\{\gamma(t): t \in \mathbb{R}\}$ is known as the moment curve in $\mathbb{R}^{2 k}$. Let $V(K)=\left\{u_{1}, \ldots, u_{n}\right\}$ be the list of all vertices of $K$ (ordered according to $\leq$ ).

Lemma D.2. Let $f$ be the linear mapping of $K$ into $\mathbb{R}^{2 k}$ given on the vertex set by $f\left(u_{i}\right):=\gamma(i)$. Then $f$ is generic and $o_{f}=(-1)^{k(k-1) / 2} o_{\gamma}$, with $o_{\gamma}$ defined above Theorem $D .1^{9}$

Proof (sketch). It is well known that every set of at most $2 k+1$ points on the moment curve in $\mathbb{R}^{2 k}$ is affinely independent, and thus the mapping is generic. Considering disjoint $k$-dimensional simplices $\sigma, \tau \in K$ and using Gale's evenness condition [Zie94, Theorem 0.7], it is easy to see that $f(\sigma) \cap f(\tau)=\emptyset$ unless the vertices of $\sigma$ and of $\tau$ alternate along the moment curve, in which case $f(\sigma)$ and $f(\tau)$ intersect (in a single point).

It remains to determine the sign of $f(\sigma) \cdot f(\tau)$, which equals the sign of the determinant of the matrix (written as a list of columns)

$$
\begin{array}{r}
{\left[\gamma\left(i_{1}\right)-\gamma\left(i_{0}\right), \gamma\left(i_{2}\right)-\gamma\left(i_{0}\right), \ldots, \gamma\left(i_{k}\right)-\gamma\left(i_{0}\right), \gamma\left(j_{1}\right)-\gamma\left(j_{0}\right), \gamma\left(j_{2}\right)-\gamma\left(j_{0}\right), \ldots,\right.} \\
\left.\gamma\left(j_{k}\right)-\gamma\left(j_{0}\right)\right]
\end{array}
$$

\footnotetext{
9 We note that if $o_{f} \in \mathfrak{o}_{K}$ then $-o_{f} \in \mathfrak{o}_{K}$ as well, since we can compose $f$ with a linear orientation-reversing map $\mathbb{R}^{2 k} \rightarrow \mathbb{R}^{2 k}$. Hence the $(-1)^{k(k-1) / 2}$ factor does not matter for the definition.
} 
for some $i_{0}<j_{0}<i_{i}<j_{1}<\cdots<i_{k}<j_{k}$. If we permute the columns to the ordering $\gamma\left(i_{1}\right)-\gamma\left(i_{0}\right), \gamma\left(j_{1}\right)-\gamma\left(j_{0}\right), \gamma\left(i_{2}\right)-\gamma\left(i_{0}\right), \ldots, \gamma\left(j_{k}\right)-\gamma\left(j_{0}\right)$, the sign is multiplied

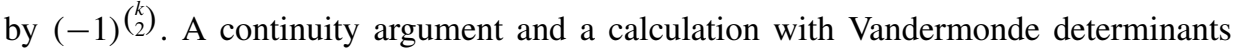
show that for any choice of reals $x_{0}<y_{0}<x_{1}<y_{1}<\cdots<x_{k}<y_{k}$ the sign of $\operatorname{det}\left[\gamma\left(x_{1}\right)-\gamma\left(x_{0}\right), \gamma\left(y_{1}\right)-\gamma\left(y_{0}\right), \ldots, \gamma\left(x_{k}\right)-\gamma\left(x_{0}\right), \gamma\left(y_{k}\right)-\gamma\left(y_{0}\right)\right]$ is always +1 .

The lemma just proved gives a geometric meaning to the definition of $o_{\gamma}{ }^{10}$ It remains to explain where the finger move vectors come from. We will also use this opportunity to outline the main ideas in the proof of Theorem D.1] (see [FKT94] for an insightful presentation of the proof).

To this end, we first extend the definition of $o_{f}$ to a generic PL mapping $f$ of $K$ into $\mathbb{R}^{2 k}$. Let $K^{\prime}$ be a subdivision of $K$ such that $f$ is linear on $K$. Then we extend the definition of the intersection number $f(\sigma) \cdot f(\tau)$ by

$$
f(\sigma) \cdot f(\tau)=\sum_{\sigma^{\prime} \triangleleft \sigma, \tau^{\prime} \triangleleft \tau} f\left(\sigma^{\prime}\right) \cdot f\left(\tau^{\prime}\right)
$$

where the sum is over all pairs $\left(\sigma^{\prime}, \tau^{\prime}\right)$ such that $\sigma^{\prime}$ is a $k$-dimensional simplex of $K^{\prime}$ contained in $\sigma$ and $\tau^{\prime}$ is a $k$-dimensional simplex of $K^{\prime}$ contained in $\tau^{11}$ Then $o_{f}$ is again defined by 3 .

Van Kampen's approach to the proof of Theorem D.1 is based on the following geometric claim: Given any two generic PL maps $f, g$ of $K$ into $\mathbb{R}^{2 k}$, there is a finite sequence $f_{0}=f, f_{1}, f_{2}, \ldots, f_{t}=g$ of generic PL maps of $K$ into $\mathbb{R}^{2 k}$ such that $f_{i+1}$ differs from $f_{i}$ only by a suitably defined elementary move (somewhat analogous to the Reidemeister moves in knot theory). Then he analyzes the effect of the various kinds of elementary moves on $o_{f}$; it turns out that the only kind of move that can possibly affect $o_{f}$ is a finger move.

To describe a finger move, we consider some generic PL mapping $f$ and two disjoint simplices $\omega, v \in K$ with $\operatorname{dim} \omega=k$ and $\operatorname{dim} v=k-1$. The finger move corresponding to $\omega$ and $v$ modifies $f$ by pulling a thin "finger" from $f(\omega)$ that wraps around $f(v)$; see Fig. 16 for an example with $k=1$. The finger can be chosen so that the modified $f$ is again a generic PL mapping.

A careful analysis of the signs of the newly introduced intersections shows that the finger move changes $o_{f}$ by one of the vectors $\varphi^{\omega, \nu},-\varphi^{\omega, \nu}$, which explains the definition of $\varphi^{\omega, v}$ given above. Since any generic PL mapping of $K$ into $\mathbb{R}^{2 k}$ can be transformed

\footnotetext{
10 Interestingly, interpreting $\mathfrak{o}_{K}$ cohomologically and expressing it as a cup product of a onedimensional cohomology class, one naturally arrives at the same element $o_{\gamma} \in \mathfrak{o}_{K}$.

11 The definition of $f(\sigma) \cdot f(\tau)$ for a linear mapping assumes a fixed linear ordering of the vertices of $K$, which we do not have for the subdivision $K^{\prime}$. Here it helps to look at $K$ and $K^{\prime}$ geometrically, assuming that we deal with a geometric realization of $K$ in some $\mathbb{R}^{m}$. Each $k$-dimensional simplex $\sigma=\left[v_{0}, v_{1}, \ldots, v_{k}\right] \in K$ spans a $k$-dimensional affine subspace $U$ of $\mathbb{R}^{m}$, and each $\sigma^{\prime} \triangleleft \sigma$ spans the same subspace. We can thus choose a linear ordering $\left[v_{0}^{\prime}, v_{1}^{\prime}, \ldots, v_{k}^{\prime}\right]$ of the vertices of $\sigma^{\prime}$ such that the bases $\left(v_{1}-v_{0}, \ldots, v_{k}-v_{0}\right)$ and $\left(v_{1}^{\prime}-v_{0}^{\prime}, \ldots, v_{k}^{\prime}-v_{0}^{\prime}\right)$ of $U$ have the same orientation. This does not necessarily lead to a linear ordering of the whole vertex set of $K^{\prime}$, but the above definition of $f(\sigma) \cdot f(\tau)$ needs only that the vertex sets $\sigma$ and $\tau$ are linearly ordered, and so we can choose a linear ordering for each $\sigma^{\prime} \in K^{\prime}$ separately.
} 

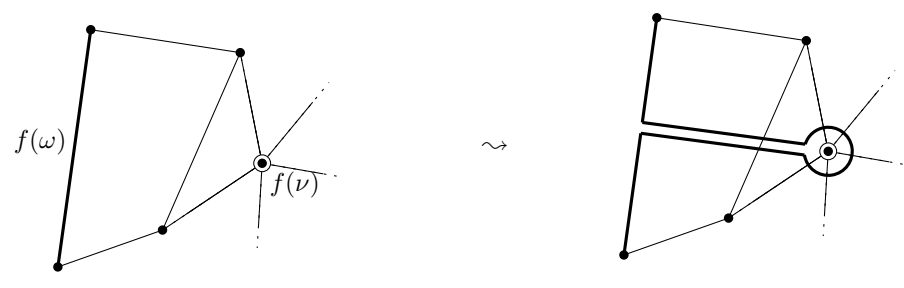

Fig. 16. A finger move.

into any other by a sequence of elementary moves (this statement we have not proved, or even properly defined, of course, but it is not difficult), it follows that $o_{f} \in \mathfrak{o}_{K}$ for all $f$. In particular, if $f$ is an embedding, we obviously have $o_{f}=0$, and thus $\mathfrak{o}_{K}$ vanishes for embeddable $K$. This yields one implication in Theorem D.1

For the reverse (and harder) implication, we suppose that $\mathfrak{o}_{K}$ vanishes, which means that $o_{\gamma}$ can be expressed as an integer linear combination of finger move vectors. This means that the generic linear mapping of $K$ into $\mathbb{R}^{2 k}$ corresponding to $o_{\gamma}$ can be transformed by a sequence of finger moves to a generic PL mapping $g$ with $o_{g}=0$. As is illustrated in Fig. 17, such a $g$ is typically not yet an embedding, since the images of nondisjoint simplices may intersect, and also the images of disjoint simplices may have intersections-we only know that the number of intersections always combines to 0 algebraically (taking the signs into account). One then needs a way of removing these two kinds of intersections. There are three kinds of elementary moves (different from finger moves) that allow one to remove such intersections; one of them is well known in topology as the Whitney trick, and the others are similar. They are guaranteed to work only for $k \geq 3$. (The graph case $k=1$ has to be treated separately and it works for a different reason 12

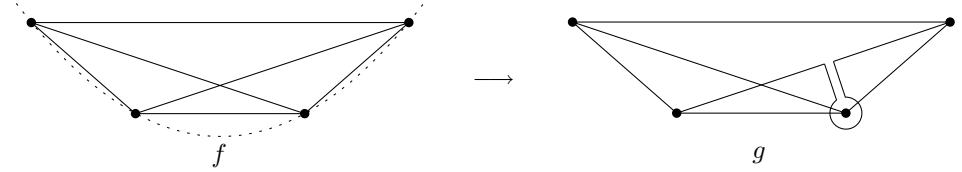

Fig. 17. A mapping $f$ of the graph $K_{4}$ into $\mathbb{R}^{2}$, with vertices on the moment curve; a finger move transforms it to a mapping $g$ with $o_{g}=0$.

Acknowledgments. We would like to thank Colin Rourke for explanations concerning PL topology and for examples showing the difference between PL embeddability and topological embeddability mentioned in Section 2 We also thank Michael Joswig, Gil Kalai, Frank Lutz, Alexander Nabutovsky, and Robin Thomas for kindly answering our questions. The second author would also like

\footnotetext{
12 For graphs this step follows from the Hanani-Tutte theorem [CH34, Tut70], which asserts that a graph that can be drawn with any two vertex-disjoint edges intersecting an even number of times is planar. In our language, this says precisely that if the Van Kampen obstruction modulo 2 vanishes, then the graph is embeddable.
} 
to thank Sergio Cabello for helpful discussions regarding linear-time algorithms for $\mathrm{EMBED}_{2 \rightarrow 2}$. Finally, we are grateful to two anonymous referees for careful reading and valuable suggestions.

Research of U. Wagner was supported by the Swiss National Science Foundation (SNF Projects 200021-116741 and 200021-125309).

\section{References}

[ABB90] Acquistapace, F., Benedetti, R., Broglia, F.: Effectiveness-noneffectiveness in semialgebraic and PL geometry. Invent. Math. 102, 141-156 (1990) Zbl 0729.14040 MR 1069244

[AHT06] Agol, I., Hass, J., Thurston, W.: The computational complexity of knot genus and spanning area. Trans. Amer. Math. Soc. 358, 3821-3850 (2006) Zbl 1098.57003 MR 2219001

[Ani89] Anick, D. J.: The computation of rational homotopy groups is \# $\wp$-hard. In: Computers in Geometry and Topology (Chicago, IL, 1986), Lecture Notes in Pure Appl. Math. 114, Deker, 1-56 (1989) Zbl 0691.55009 MR 0988689

[AB09] Arora, S., Barak, B.: Complexity Theory: A Modern Approach. Cambridge Univ. Press, Cambridge (2009); http://www.cs.princeton.edu/theory/complexity/ Zbl 1193.68112 MR 2500087

[Bin59] Bing, R. H.: An alternative proof that 3-manifolds can be triangulated. Ann. of Math. (2) 69, 37-65 (1959) Zbl 0106.16604 MR 0100841

[Böh90] Böhme, T.: On spatial representations of graphs. In: Contemporary Methods in Graph Theory, Bibliogr. Inst., Mannheim, 151-167 (1990) Zbl 0718.05054 MR 1126225

[BGdO00] Bokowski, J., Guedes de Oliveira, A.: On the generation of oriented matroids. Discrete Comput. Geom. 24, 197-208 (2000) Zbl 0969.52008 MR 1756651

[Bre83] Brehm, U.: A nonpolyhedral triangulated Möbius strip. Proc. Amer. Math. Soc. 89, 519-522 (1983) Zbl 0526.57013 MR 0715878

[BS92] Brehm, U., Sarkaria, K. S.: Linear vs. piecewise linear embeddability of simplicial complexes. Tech. Report 92/52, Max-Planck-Institut f. Mathematik, Bonn (1992)

[Bro57] Brown, E. H. (jun.): Finite computability of Postnikov complexes. Ann. of Math. (2) 65, 1-20 (1957) Zbl 0077.16804 MR 0083733

[Bry72] Bryant, J. L.: Approximating embeddings of polyhedra in codimension three. Trans. Amer. Math. Soc. 170, 85-95 (1972) Zbl 0259.57007 MR 0307245

[Bry02] Bryant, J. L.: Piecewise linear topology. In: R. J. Daverman et al. (eds.), Handbook of Geometric Topology, Elsevier, Amsterdam, 219-259 (2002) Zbl 0986.57021 MR 1886671

[Buo03] Buoncristiano, S.: Fragments of Geometric Topology from the Sixties. Geom. Topol. Monogr. 6 (2003). New edition in preparation, in collaboration with C. Rourke Zbl 1066.57001

[CH34] Chojnacki (Hanani), C.: Über wesentlich unplättbare Kurven im dreidimensionalen Raume. Fund. Math. 23, 135-142 (1934) Zbl 0009.41104

[CZ61] Curtis, M. L., Zeeman, E. C.: On the polyhedral Schoenflies theorem. Proc. Amer. Math. Soc. 11, 888-889 (1961) Zbl 0096.37903 MR 0119208

[DSV01] Dumas, J.-G., Saunders, B. D., Villard, G.: On efficient sparse integer matrix Smith normal form computations. J. Symbolic Comput. 32, 71-99 (2001) Zbl 1050.65044 MR 1840386

[Flo34] Flores, A.: Über $n$-dimensionale Komplexe, die im $R_{2 n+1}$ absolut selbstverschlungen sind. Ergeb. Math. Kolloq. 6, 4-7 (1932/1934) Zbl 0011.03804 
[Fre82] Freedman, M. H.: The topology of four-dimensional manifolds. J. Differential Geom. 17, 357-453 (1982) Zbl 0528.57011 MR 0679066

[FKT94] Freedman, M. H., Krushkal, V. S., Teichner, P.: Van Kampen's embedding obstruction is incomplete for 2-complexes in $\mathbb{R}^{4}$. Math. Res. Lett. 1, 167-176 (1994) Zbl 0847.57005 MR 1266755

[Gie01] Giesbrecht, M.: Fast computation of the Smith form of a sparse integer matrix. Comput. Complexity 10, 41-69 (2001) Zbl 0992.65039 MR 1867308

[Gla71] Glaser, L. C.: A proof of the most general polyhedral Schoenflies conjecture possible. Pacific J. Math. 38, 401-417 (1971) Zbl 0194.55604 MR 0312513

[Gol08] Goldreich, O.: Computational Complexity: A Conceptual Perspective. Cambridge Univ. Press, Cambridge (2008); http://www.wisdom.weizmann.ac.il/ oded/ccbook.html Zbl 1154.68056 MR 2400985

[GS06] Gonçalves, D., Skopenkov, A.: Embeddings of homology equivalent manifolds with boundary. Topology Appl. 153, 2026-2034 (2006) Zbl 1105.57022 MR 2237594

[Hae64] Haefliger, A.: Plongements de variétés dans le domaine stable. In: Sém. Bourbaki 1962/63, fasc. 1, exp. 245 (1964) Zbl 0127.13701 MR 0189049 MR 1611531

[Hak61] Haken, W.: Theorie der Normalflächen. Acta Math. 105, 245-375 (1961) Zbl 0100.19402 MR 0141106

[HJ64] Halin, R., Jung, H. A.: Charakterisierung der Komplexe der Ebene und der 2-Sphäre. Arch. Math. 15, 466-469 (1964) Zbl 0125.11604 MR 0170341

[HLP99] Hass, J., Lagarias, J. C., Pippenger, N.: The computational complexity of knot and link problems. J. ACM 46, 185-211 (1999) Zbl 1065.68667 MR 1693203

[Hat01] Hatcher, A.: Algebraic Topology. Cambridge Univ. Press, Cambridge (2001); http://math.cornell.edu/hatcher\#AT1 Zbl 1044.55001 MR 1867354

[Hem79] Hemion, G.: On the classification of homeomorphisms of 2-manifolds and the classification of 3-manifolds. Acta Math. 142, 123-155 (1979) Zbl 0402.57027 MR 0512214

[HT74] Hopcroft, J., Tarjan, R.: Efficient planarity testing. J. ACM 21, 549-568 (1974) Zbl 0307.68025 MR 0359387

[Iva08] Ivanov, S. V.: The computational complexity of basic decision problems in 3-dimensional topology. Geom. Dedicata 131, 1-26 (2008) Zbl 1146.57025 MR 2369189

[Lic65] Lickorish, W. B. R.: The piecewise linear unknotting of cones. Topology 4, 67-91 (1965) Zbl 0138.19003 MR 0203736

[MS66] Mardešić, S., Segal, J.: A note on polyhedra embeddable in the plane. Duke Math. J. 33, 633-638 (1966) Zbl 0168.21602 MR 0199848

[MS67] Mardešić, S., Segal, J.: $\varepsilon$-mappings and generalized manifolds. Michigan Math. J. 14, 171-182 (1967) Zbl 0152.21704 MR 0211407

[Mar58] Markov, A. A.: The insolubility of the problem of homeomorphy. Dokl. Akad. Nauk SSSR 121, 218-220 (1958) (in Russian) Zbl 0092.00702 MR 0097793

[Mat03] Matoušek, J.: Using the Borsuk-Ulam Theorem. Springer, Berlin (2003) Zbl 1016.05001 MR 1988723

[Mat97] Matveev, S. V.: Classification of sufficiently large 3-manifolds. Uspekhi Mat. Nauk 52, no. 5, 147-174 (1997) (in Russian); English transl.: Russian Math. Surveys 52, no. 5, 1029-1055 (1997) Zbl 0916.57019 MR 1490029

[Mel06] Melikhov, S. A.: The van Kampen obstruction and its relatives. Tr. Mat. Inst. Steklova 266, 149-183 (2009) Zbl pre05655906 MR 2603266

[Mun84] Munkres, J. R.: Elements of Algebraic Topology. Addison-Wesley, Reading, MA (1984) Zbl 0673.55001 MR 0755006 
[Nab95] Nabutovsky, A.: Einstein structures: Existence versus uniqueness. Geom. Funct. Anal. 5, 76-91 (1995) Zbl 0842.53032 MR 1312020

[NW96] Nabutovsky, A., Weinberger, S.: Algorithmic unsolvability of the triviality problem for multidimensional knots. Comment. Math. Helv. 71, 426-434 (1996) Zbl 0862.57017 MR 1418946

[NW99] Nabutovsky, A., Weinberger, S.: Algorithmic aspects of homeomorphism problems. In: Tel Aviv Topology Conference: Rothenberg Festschrift (1998), Contemp. Math. 231, Amer. Math. Soc., Providence, RI, 245-250 (1999) Zbl 0932.57021 MR 1707346

[New60] Newman, M. H. A.: On the division of Euclidean $n$-space by topological $(n-1)$ spheres. Proc. Roy. Soc. London Ser. A 257, 1-12 (1960) Zbl 0094.17601 MR 0123305

[Pap43] Papakyriakopoulos, C.: A new proof for the invariance of the homology groups of a complex. Bull. Soc. Math. Grèce 22, 1-154 (1943) (in Greek) MR 0024619

[Ren92] Renegar, J.: On the computational complexity and geometry of the first-order theory of the reals. I, II, III. J. Symbolic Comput. 13, 255-299, 301-327, 329-352 (1992) Zbl 0763.68042(Vol. I), Zbl 0763.68043(Vol. II), Zbl 0798.68073(Vol. III) MR 1156882(Vol. I), MR 1156883(Vol. II), MR 1156884(Vol. III).

[RST93] Robertson, N., Seymour, P. D., Thomas, R.: A survey of linkless embeddings. In: Graph Structure Theory (Seattle, WA, 1991), Contemp. Math. 147, Amer. Math. Soc., Providence, RI, 125-136 (1993) Zbl 0788.05034 MR 1224699

[RST95] Robertson, N., Seymour, P. D., Thomas, R.: Sachs' linkless embedding conjecture. J. Combin. Theory Ser. B 64, 185-227 (1995) Zbl 0832.05032 MR 1339849

[RRS06] Romero, A., Rubio, J., Sergeraert, F.: Computing spectral sequences. J. Symbolic Comput. 41, 1059-1079 (2006) Zbl 1132.55008 MR 2262083

[RS82] Rourke, C. P., Sanderson, B. J.: Introduction to Piecewise-Linear Topology. Springer, Berlin (1982) Zbl 0477.57003 MR 0350744

[Rub95] Rubinstein, J. H.: An algorithm to recognize the 3-sphere. In: Proc. Int. Congress of Mathematicians, Vol. 1, 2 (Zürich, 1994), Birkhäuser, Basel, 601-611 (1995) Zbl 0864.57009 MR 1403961

[Sch04] Schleimer, S.: Sphere recognition lies in NP. http://www.warwick.ac.uk/ masgar (2004)

[Sch91] Schön, R.: Effective algebraic topology. Mem. Amer. Math. Soc. 92, no. 451 (1991) Zbl 0731.55015 MR 1055531

[SSS98] Segal, J., Skopenkov, A., Spież, S.: Embeddings of polyhedra in $\mathbb{R}^{m}$ and the deleted product obstruction. Topology Appl. 85, 335-344 (1998) Zbl 0934.57025 MR 1617472

[SS92] Segal, J., Spież, S.: Quasi embeddings and embeddings of polyhedra in $\mathbb{R}^{m}$. Topology Appl. 45, 275-282 (1992) Zbl 0768.57012 MR 1180814

[Sha57] Shapiro, A.: Obstructions to the imbedding of a complex in a euclidean space. I: The first obstruction. Ann. of Math. (2) 66, 256-269 (1957) Zbl 0085.37701 MR 0089410

[Sko06] Skopenkov, A.: Embedding and knotting of manifolds in euclidean spaces. In: London Math. Soc. Lecture Note Ser. 347, 248-342 (2008) Zbl 1154.57019 MR 2388495

[Smi98] Smith, J.: m-structures determine integral homotopy type. arXiv:math/9809151v1 (1998)

[Soa04] Soare, R. I.: Computability theory and differential geometry. Bull. Symbolic Logic 10, 457-486 (2004) Zbl 1085.03033 MR 2136634

[Sta65] Stallings, J.: Homology and central series of groups. J. Algebra 2, 170-181 (1965) Zbl 0135.05201 MR 0175956 
[Ste47] Steenrod, N. E.: Products of cocycles and extensions of mappings. Ann. of Math. (2) 48, 290-320 (1947) Zbl 0030.41602 MR 0022071

[Sto96] Storjohann, A.: Near optimal algorithms for computing Smith normal forms of integer matrices. In: International Symposium on Symbolic and Algebraic Computation (Zürich, 1996), ACM Press, New York, 267-274 (1996) Zbl 0914.65043

[Tho94] Thompson, A.: Thin position and the recognition problem for $S^{3}$. Math. Res. Lett. 1, 613-630 (1994) Zbl 0849.57009 MR 1295555

[Tut70] Tutte, W. T.: Toward a theory of crossing numbers. J. Combin. Theory 8, 45-53 (1970) Zbl 0187.20803 MR 0262110

[vK32] van Kampen, E. R.: Komplexe in euklidischen Räumen. Abh. Math. Sem. Hamburg 9, 72-78 (1932); Berichtigung, ibid., 152-153 JFM 58.0615.02 JFM 58.0615.03

[VKF74] Volodin, I. A., Kuznetsov, V. E., Fomenko, A. T.: The problem of discriminating algorithmically the standard three-dimensional sphere. Uspekhi Mat. Nauk 29, no. 5, 71-168 (1974) (in Russian); English transl.: Russian Math. Surveys 29, no. 5, 71-172 (1974) Zbl 0311.57001 MR 0405426

[Web67] Weber, C.: Plongements de polyèdres dans le domaine metastable. Comment. Math. Helv. 42, 1-27 (1967) Zbl 0152.22402 MR 0238330

[Wig07] Wigderson, A.: P, NP and Mathematics-a computational complexity perspective. In: Proc. ICM 06 (Madrid), Vol. 1, Eur. Math. Soc. Publ. House, Zürich, 665-712 (2007); Zbl 1149.68033 MR 2334207

[Wu65] Wu, W.-T.: A Theory of Imbedding, Immersion, and Isotopy of Polytopes in a Euclidean Space. Science Press, Peking (1965) Zbl 0177.26402

[Zie94] Ziegler, G. M.: Lectures on Polytopes. Springer, Berlin (1995) Zbl 0823.52002 MR 1311028 